\title{
TU/e emonownen

\section{Modelling traffic flows and estimating road travel times in transportation network under dynamic disturbances}

\section{Citation for published version (APA):}

Jiang, J., Dellaert, N. P., van Woensel, T., \& Wu, L. (2020). Modelling traffic flows and estimating road travel times in transportation network under dynamic disturbances. Transportation, 47(6), 2951-2980.

https://doi.org/10.1007/s11116-019-09997-3

\section{Document license:}

TAVERNE

DOI:

10.1007/s11116-019-09997-3

Document status and date:

Published: 01/12/2020

\section{Document Version:}

Publisher's PDF, also known as Version of Record (includes final page, issue and volume numbers)

\section{Please check the document version of this publication:}

- A submitted manuscript is the version of the article upon submission and before peer-review. There can be important differences between the submitted version and the official published version of record. People interested in the research are advised to contact the author for the final version of the publication, or visit the $\mathrm{DOI}$ to the publisher's website.

- The final author version and the galley proof are versions of the publication after peer review.

- The final published version features the final layout of the paper including the volume, issue and page numbers.

Link to publication

\section{General rights}

Copyright and moral rights for the publications made accessible in the public portal are retained by the authors and/or other copyright owners and it is a condition of accessing publications that users recognise and abide by the legal requirements associated with these rights.

- Users may download and print one copy of any publication from the public portal for the purpose of private study or research.

- You may not further distribute the material or use it for any profit-making activity or commercial gain

- You may freely distribute the URL identifying the publication in the public portal.

If the publication is distributed under the terms of Article 25fa of the Dutch Copyright Act, indicated by the "Taverne" license above, please follow below link for the End User Agreement:

www.tue.nl/taverne

Take down policy

If you believe that this document breaches copyright please contact us at:

openaccess@tue.nl

providing details and we will investigate your claim. 


\title{
Modelling traffic flows and estimating road travel times in transportation network under dynamic disturbances
}

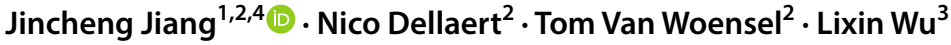 \\ Published online: 1 April 2019 \\ (c) Springer Science+Business Media, LLC, part of Springer Nature 2019
}

\begin{abstract}
Traffic congestion is a common phenomenon in road transportation networks, especially during peak hours. More accurate prediction of dynamic traffic flows is very important for traffic control and management. However, disturbances caused by the time-varying origindestination matrix, dynamic route choices, and disruptions make the modelling of traffic flows difficult. Therefore, this study focuses on modelling the dynamic evolution processes of traffic flows under disturbances and estimating dynamic travel times for arbitrary moment. A revised Lighthill-Whitham-Richards (RLWR) model with non-equilibrium states is presented to describe the dynamic traffic states on individual roads, and the ripplespreading model (RSM) is integrated to investigate the interactions among several shockwaves from multiple roads. We propose a hybrid RLWR-RSM to model the congestion and congestion-recovery propagations in an entire transportation network. After predicting the dynamic traffic flows by the RLWR-RSM, the road travel times for arbitrary moment were estimated. Theoretical analyses indicated that (1) the RLWR-RSM inherits the advantages of macroscopic traffic flow models and integrates the characteristics of both low- and highorder continuum models, and (2) the RLWR-RSM considers multiple disturbances. From numerical experiments with various inputs, the variation in travel times under disturbances was investigated, and this further demonstrated that (1) the modelled dynamic traffic flows have four basic properties, and (2) the experimental results validate the theoretical analyses. In addition, the RLWR-RSM can explain several distinct traffic phenomena. Finally, the estimated travel times can provide decision supports for vehicle navigation.
\end{abstract}

Keywords Traffic flow $\cdot$ Disturbance $\cdot$ Time-varying OD $\cdot$ Route choice $\cdot$ Network disruption $\cdot$ Dynamic travel time

Jincheng Jiang

j.jiang@szu.edu.cn

1 Shenzhen Key Laboratory of Spatial Information Smart Sensing and Services, School of Architecture and Urban Planning, Research Institute for Smart Cities, Shenzhen University, Shenzhen 518060, China

2 School of Industrial Engineering and Innovation Sciences, Technical University of Eindhoven, P.O. Box 513, Eindhoven 5600 MB, Netherlands

3 School of Geoscience and Info-Physics, Central South University, Changsha 410083, China

4 Shenzhen Institutes of Advanced Technology, Chinese Academy of Sciences, Shenzhen 518055, China 


\section{Introduction}

Traffic prediction (Matas et al. 2012) is important for the shortest path (Fu and Rilett 1998; Sever et al. 2013), traffic assignment (Peeta and Ziliaskopoulos 2001), disaster emergency (Jiang and Wu 2017; Jiang et al. 2017), and vehicle routing (Kim et al. 2005). However, traffic states are dynamic, which makes the prediction of travel times difficult (Fu 2002). Many methods have been proposed to address this issue, such as statistical analyses, surveillance systems (Turner 1996), discrete-time Markov chain models (Yeon et al. 2008), and artificial neural network models (Lnnamaa 2005). Among such methods, whole-link models (Carey and Ge 2003; Carey et al. 2003), which estimate dynamic road travel times by comparing cumulative inflows and outflows, are representative. A drawback of these models is the absence of a description of the dynamic congestion propagation processes. Thus, they cannot describe rapidly changing traffic flows accurately (Carey et al. 2003). To overcome this, a precise description of the relationship among the dynamic flows, vehicle density, and velocities is necessary (Kockelman 2001). For this purpose, dynamic traffic flow models are a good candidate.

Traffic flows are dynamic processes (Daganzo 1994a, 1995b), and they significantly influence many applications (Gentile et al. 2007; Sheu et al. 2001; Papageorgiou et al. 2003). Traffic flow models are generally classified as macroscopic, mesoscopic, and (sub-) microscopic models (Hoogendoorn and Bovy 2001); all of these models provide respective advantages and disadvantages. In this study, a macroscopic model is chosen as the theoretical basis because of its distinct advantages: (1) it accurately and directly describes the relationship among macroscopic variables and provides improved results for some scenarios; (2) only a small amount of easily observable and measured data is required to reliably describe the congestion and congestion-recovery propagations using mathematical analysis; (3) it is applicable for large-scale macroscopic traffic simulation, analysis, and reproduction because less computational time is consumed due to the elimination of the need to consider the individual behaviours of all vehicles (Hoogendoorn and Bovy 2001).

Macroscopic models are indispensable in modelling dynamic traffic flow because they accurately and concisely describe macroscopic variables such as density, velocity, and flow rate. In addition to the 'hydrodynamic' flow-density relation and differential equation (Treiber and Kesting 2014), which were derived from the assumption of continuum of traffic flows (Jin 1977), both low- and high-order continuum relationships of macroscopic variables are presented: (1) In the low-order continuum Lighthill-Whitham-Richards (LWR) model (Lighthill and Whitham 1955; Richards 1956), velocity is an explicit function of density, which is valid for stationary states. The LWR model captures the characteristics of a car-following model at an aggregate level (Newell 1961) and is consistent with flow shockwave models (Whitham and Fowler 2008). However, Liu et al. (1998) indicated that this model cannot describe the amplification of small disturbances in heavy traffic. The cell transmission model (CTM) (Daganzo 1994b, 1995a) is an important model for macroscopic network traffic flows. It is a discretisation of the LWR model and can completely describe congestion formation, spreading, and elimination. However, the CTM records the dynamic vehicle density for all road cells in each time moment, resulting in a high computational time and storage requirements. (2) In high-order continuum models, Payne-type models Payne $(1971,1979)$ have been developed using relaxation time and anticipation coefficients. They describe the decrease rate of equilibrium velocities with increasing density. By considering the effects of acceleration and deceleration, Payne-type models overcome the drawbacks of the LWR model. However, Daganzo (1995b) showed that negative 
flows and velocities may be predicted by the Payne-type models under certain conditions. In addition, a few other high-order models have been developed Michalopoulos et al. (1992); Helbing (1996); Papageorgiou et al. (2003). More detailed reviews and comparisons of macroscopic traffic flow models can be found in Hoogendoorn and Bovy (2001); Treiber and Kesting (2014).

In this study, we developed a revised Lighthill-Whitham-Richards (RLWR) model, which considers a vehicle's acceleration and deceleration behaviours. In addition, the disturbances caused by the time-varying origin-destination (OD) matrix (Castillo et al. 2013), dynamic route choices (Xiong et al. 2015), and disruptions are modelled. Furthermore, their effects on both individual roads and an entire transportation network are analysed using the ripple-spreading model (RSM). Thus, the propagations of congestion and recovery for an entire network are modelled. Finally, the road travel times (Xu et al. 2013) at arbitrary moments are estimated.

The following are the main contributions of this paper:

(1) In order to overcome the drawbacks of both low- and high-order continuum models, we propose the RLWR model, which inherits the advantages of both LWR and Payne-type models. The RLWR model not only considers vehicle velocity as an explicit function of density but also considers the acceleration and deceleration processes.

(2) Considering the disturbances caused by the dynamic OD matrix, disruptions, and route choices, a hybrid RLWR-RSM, which integrates the RLWR model and RSM, is proposed to model the dynamic traffic flows on individual roads and to further investigate the interaction among dynamic traffic flows of multiple roads. The hybrid RLWR-RSM has four basic properties namely first-in-first-out, reduction to static model, causality, and flow conversation. Moreover, this model is used to explain distinct traffic flow phenomena.

(3) With known dynamic processes of traffic flows, the road travel times at arbitrary time moments are estimated with consideration of the propagation of congestion and recovery.

The remainder of this paper is organised as follows: The required definitions and formulations are provided in Sect. 2. The conventional LWR model and RSM are introduced in Sect. 3. The hybrid RLWR-RSM is presented in Sect. 4. Section 5 discusses the modelling of three kinds of disturbances on traffic flows and provides an estimation method for road travel times. Experiments are described and discussions on dynamic traffic flows and road travel times are presented in Sect. 6. We present conclusions by providing an overview of results and future research hotspots in "Acknowledgements".

\section{Definitions}

This section provides the definitions of several key concepts and notations used in this paper.

The transportation network is modelled as a directed network $G(N, A) . n=|N|$ represents the number of road intersections and $m=|A|$ represents the number of roads, where $N$ is the intersection set, and $A$ is the road set. Each $\operatorname{road}(i, j) \in A$ is associated with length $l_{i j}$, capacity $u_{i j}$, vehicle velocity $v_{i j}(x, t)$, vehicle density $\rho_{i j}(x, t)$, and vehicle flow rate $q_{i j}(x, t)$, where $x$ and $t$ 
are the space location and time moment, respectively. Velocity, density, and flow rate are considered as the basic macroscopic traffic flow variables in traffic flow theory.

Let $R_{k}\left(E_{p}, T_{t}, T_{s}, E_{s}, E_{u}, E_{d}, v, I_{u}, I_{d}\right)$ be a ripple, which is considered as a shockwave in traffic flow theory, where $E_{p}$ is the epicentre coordinate, $T_{t}$ is the triggering time, $T_{s}$ is current time, $E_{s}$ is current location, $E_{u}$ and $E_{d}$ are the energies (or vehicle densities) at the upstream and downstream sides, respectively, $v$ is the shockwave speed $(v>0$ if it moves downstream; $v<0$ if it moves upstream; otherwise, $v=0$ if it does not move), and $I_{u}$ and $I_{d}$ are the vehicle information (e.g. destinations and path choices) at the upstream and downstream sides, respectively.

$$
M_{O D}(t)=\left[\begin{array}{ccc}
S_{1,1}(t) & \cdots & S_{1, n}(t) \\
\vdots & \ddots & \vdots \\
S_{n, 1}(t) & \cdots & S_{n, n}(t)
\end{array}\right]
$$

shows the predictable time-varying OD matrix for the forthcoming hours, where $S_{i j}(t)$ denotes the vehicle flow rate from node $i$ to node $j$ at time moment $t$. On an arbitrary individual road $(i, j) \in A$, the inflow rate $I_{i j}(t)$ and outflow rate $O_{i j}(t)$ are dynamic.

$P_{i, j}^{k}$ is the $k$ th $(1 \leqslant k \leqslant w)$ driving path choice from node $i$ to $j$, where $w$ is the total number of available path choices. $R_{r}\left(I_{u}\right)=\left\{\left(F_{1}, D_{1}, P_{1}\right), \cdots,\left(F_{w}, D_{w}, P_{w}\right)\right\}$ is the upstream vehicle information of shockwave $R_{r}$ on $\operatorname{road}(i, j) \in A$, where $F_{k}$ is the vehicle flow rate (this is similar to $\left.q_{i j}(x, t)\right)$; all vehicles in $F_{k}$ move along the path $P_{k}=P_{i, D_{k}}^{k}(1 \leqslant k \leqslant w)$. The downstream vehicle information $R_{r}\left(I_{d}\right)$ is similar to that of the upstream vehicle information. Similar to $R_{r}\left(I_{u}\right)$, the inflow and outflow of $\operatorname{road}(i, j)$ at time moment $t$ are $F_{i j}^{i n}(t)=\left\{\left(F_{1}^{i n}, D_{1}^{i n}, P_{1}^{i n}\right), \cdots,\left(F_{w}^{i n}, D_{w}^{i n}, P_{w}^{i n}\right)\right\}$ and $F_{i j}^{\text {out }}(t)=\left\{\left(F_{1}^{\text {out }}, D_{1}^{\text {out }}, P_{1}^{\text {out }}\right), \cdots,\left(F_{w}^{\text {out }}, D_{w}^{\text {out }}, P_{w}^{\text {out }}\right)\right\}$, respectively, where $\left(F_{k}^{\text {in }}, D_{k}^{\text {in }}, P_{k}^{\text {in }}\right)$ and $\left(F_{k}^{\text {out }}, D_{k}^{\text {out }}, P_{k}^{\text {out }}\right)$ are similar to $\left(F_{k}, D_{k}, P_{k}\right) \in R_{r}\left(I_{u}\right)$.

Let $\operatorname{Drp}\left(D_{p}, T_{p}, C_{d}, R_{c}\right)$ be a network disruption, where $D_{p}$ is the location of disruption, $T_{p}$ is occurrence time, $C_{d}$ is current capacity (i.e., the maximum flow rate for vehicles to move) after a disruption, and $R_{c}$ is recovery strength.

Generally, macroscopic traffic flow models describe dynamic density $\rho$, velocity $v$, and flow rate $q$ at a high aggregate level using partial differential equations (Hoogendoorn and Bovy 2001). Two equations are generally acknowledged by macroscopic models: the 'hydrodynamic' flow-density relation and the differential equation:

$$
\begin{aligned}
& q(x, t)=\rho(x, t) \times v(x, t) \\
& \frac{d \rho(x, t)}{d t}+\frac{d q(x, t)}{d x}=0
\end{aligned}
$$

where $x$ and $t$ are the space location and time moment, respectively.

\section{Related models}

This paper focuses on the LWR model and RSM. It is concluded from (1) and (2) that $\frac{d \rho(x, t)}{d t}+\frac{d q(x, t)}{d x}=\frac{d \rho(x, t)}{d t}+\frac{d q(x, t)}{d \rho(x, t)} \times \frac{d \rho(x, t)}{d x}=0$, and we set

$$
v_{c}=\frac{d x}{d t}=-\frac{d q(x, t)}{d \rho(x, t)}
$$


To completely describe the dynamic traffic flows, another relationship among $\rho(x, t), v(x, t)$, and $q(x, t)$ must be additionally known. The LWR model (Lighthill and Whitham 1955; Richards 1956) generally considers a traffic flow as a continuous fluid flow and uses an explicit velocity-density relationship equation, which is known as the fundamental diagram, given by

$$
v_{i j}(x, t)=V\left(\rho_{i j}(x, t)\right)
$$

This indicates that a one-to-one relationship between velocity and density (Cho and Lo 2002) exists in equilibrium states. Under Rankine-Hugoniot conditions (Hirsch 1990), an equation is available to describe the shockwave speed $\omega$ (Hoogendoorn et al. 2008), which is given as

$$
q_{2}-q_{1}=\omega \times\left(\rho_{2}-\rho_{1}\right)
$$

If $q_{2} \rightarrow q_{1}$, then $\omega=\lim _{q_{2} \rightarrow q_{1}} \frac{q\left(\rho_{2}\right)-q\left(\rho_{1}\right)}{\rho_{2}-\rho_{1}}=\frac{d q\left(\rho_{1}\right)}{d \rho}=-v_{c}\left(\rho_{1}\right)$. The LWR model along with Equations 1 and 5 provides a fundamental diagram $Q(\rho)$ (Daganzo 1994a; Gentile et al. 2007), where $v_{c}\left(\rho_{1}\right)$ is the tangent slope at a given density $\rho_{1}$, and $v_{c}$ denotes the vector of kinematic wave speed. Because it describes dynamic traffic flows using a combination of shock and rarefaction waves (Whitham and Fowler 2008), the LWR model is also called the kinematic wave model (KWM) (Jin 1977) and can be used to intuitively model the breakdown or phase-transition (Hoogendoorn et al. 2008).

The ripple-spreading model (RSM) was proposed by Hu et al. (2011); it was motivated by the natural ripple-spreading phenomenon. The main principle of this model is as follows: Some stakes are located in a quiet pond and a stone is thrown in it. An initial ripple (shockwave) is generated and spreads out. If the ripple hits a stake, it creates a new ripple because of the reflection effect. As the ripples spread, an increasing number of ripples are stimulated around the stakes. However, the shockwaves continue to weaken due to energy decay.

The RSM has two desirable advantages in modelling traffic flows: (1) The ripplespreading process embedded in the model naturally captures many real-world spatial and temporal characteristics. (2) A great flexibility is available to modify and extend the RSM (Hu et al. 2011). In order to describe the dynamic traffic flow of an entire traffic network, the RSM is associated with traffic flows as follows: (1) Stakes represent the intersections in a transportation network and stones refer to disturbances. (2) Ripples denote the shockwaves in traffic flow theory and they spread along network roads. (3) The energy of ripples is based on vehicle density. The speed of shockwaves is not constant, and it is determined by the traffic states. (4) When a shockwave $R_{u}$ reaches the entry (or exit), several responding shockwaves are simulated on the connected roads because of the change in inflows (or outflows). (5) Energy is not lost or gained when shockwaves spread. (6) Generation of new shockwaves is subject to the flow conservation constraints. (7) Shockwaves are triggered if the vehicle information or density changes. A brief dynamic process of ripple spreading in a network is illustrated in Fig. 1.

\section{Hybrid traffic flow model}

The LWR model is improved to overcome its limitations and integrated with the RSM to investigate the interaction among dynamic traffic flows of multiple roads. Based on the dynamic traffic flows predicted by this hybrid model, the road travel times can be estimated for all time moments. 


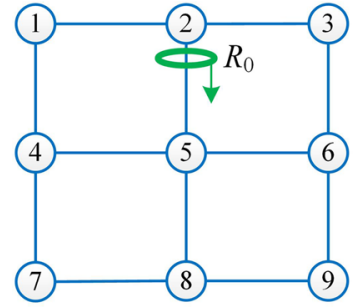

(a)

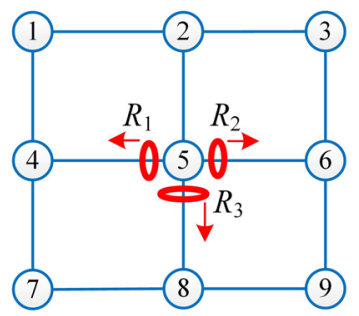

(c)

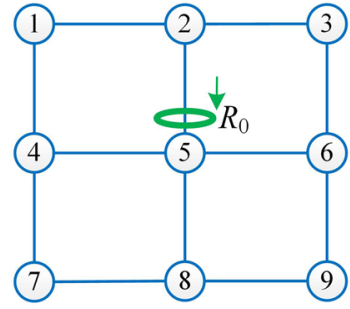

(b)

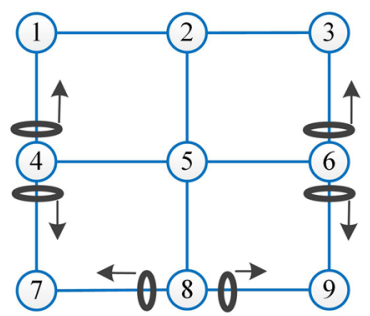

(d)
Legend:

(i) Node

Road

Shockwave

The moving direction of shockwave

Fig. 1 Mechanism of using the ripple-spreading method to model dynamic traffic flows: a The flow from node 2 to node 5 changes, and shockwave $R_{0}$ is generated; $\mathbf{b} R_{0}$ spreads along road $(2,5)$; $\mathbf{c}$ Shockwaves $R_{1}$, $R_{2}$, and $R_{3}$ are triggered, which are caused by $R_{0}$; $\mathbf{d}$ more shockwaves around nodes 4 , 6, and 8 are generated because of $R_{1}, R_{2}$, and $R_{3}$

\section{Revised Lighthill-Whitham-Richards model}

Because the flow-density relationship in the acceleration and deceleration processes does not completely follow the fundamental diagram and because the conventional LWR model cannot describe the non-equilibrium states, the LWR model can be revised to overcome its limitations by considering the dilatorily changing velocities. In this study, a buffer is considered to exist between two consecutive regions 1 and 2, for the drivers to decelerate or accelerate (Fig. 2). The flow-density relationship is not at equilibrium inside the buffer, and it follows the fundamental diagram outside the buffer. The acceleration (or deceleration) behaviour $B e(\chi)$ from $v_{1}$ to $v_{2}$ is associated with three parameters: reaction time $\Delta T_{\chi}$, acceleration time $T_{\chi}$, and acceleration strength $S_{\chi}$ (Fig. 3). The reaction time $\Delta T_{\chi}$ is the time interval for two neighbouring vehicles to change their velocities. Acceleration time $T_{\chi}$ is the time consumed in changing the velocity from $v_{1}$ to $v_{2} ; S_{\chi}=\int_{0}^{T_{\chi}}(v \times d t)-v_{2} \times T_{\chi}$.

Acceleration time and acceleration strength are always zero in the LWR model, but they are flexible variables in the revised LWR model. To reveal the relationship among the

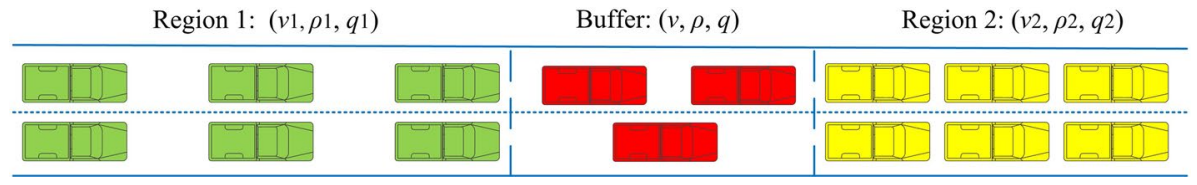

Fig. 2 The buffer between two consecutive regions, where the traffic condition $(v, \rho, q)$ are non-equilibrium because vehicles inside the buffer are accelerating or decelerating 


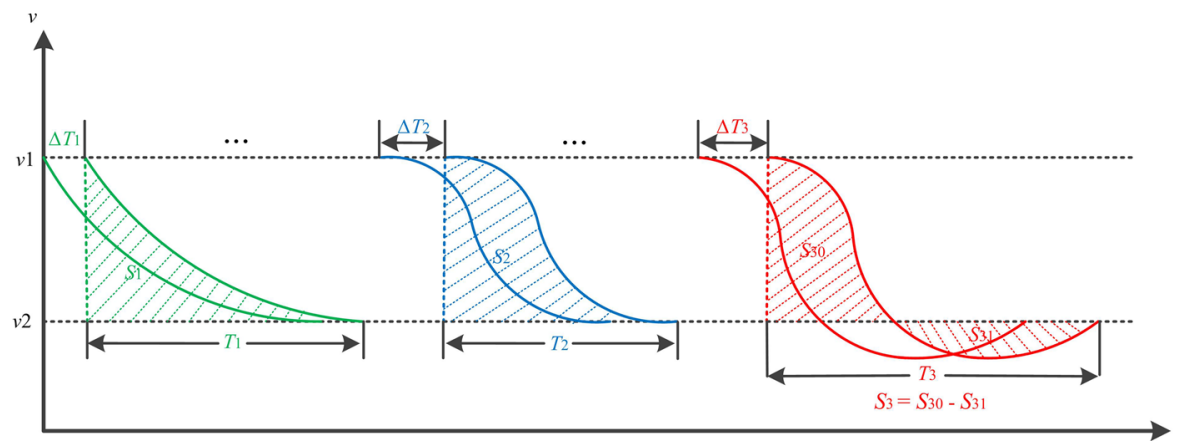

Fig. 3 The vehicle behaviors with varying parameters in a deceleration process, where solid lines denote the velocity changed from $v_{1}$ to $v_{2}$, reaction time $\Delta T_{\chi}$ means the time interval for two neighboring vehicles to change their velocities, acceleration time $T_{\chi}$ is the time consumed to change velocity from $v_{1}$ to $v_{2}$, and $S_{\chi}$ is the travel distance difference compared with that when $\chi$ travels at $v_{2}$

above three parameters, both one individual buffer and two meeting buffers are analysed. In 'one individual buffer', only one shockwave spreads without interacting with the other shockwaves; 'two meeting buffers' appear when two shockwaves interact with each other.

\section{One individual buffer}

Given the two regions $\operatorname{Re}_{1}\left(\rho_{1}, v_{1}, f_{1}\right)$ and $\operatorname{Re}_{2}\left(\rho_{2}, v_{2}, f_{2}\right)$, vehicles change their velocities from $v_{1}$ to $v_{2}$ through the buffer $B(1,2)$, and the vehicle-vehicle distance is changed from $L_{1}=\frac{1}{\rho_{1}}$ to $L_{2}=\frac{1}{\rho_{2}}$.

Theorem 1 For any two consecutive vehicles $\chi$ and $\chi+1$ in the buffer, $S_{\chi+1}-S_{\chi}=$ $\left(L_{1}-L_{2}\right)-\left(v_{1}-v_{2}\right) \times \Delta T_{\chi+1}$.

Proof Suppose vehicle $\chi$ starts changing its velocity at time moment $t_{0}$. (1) For the vehicle $\chi$, the driving distance $d_{\chi, 1}=v_{2} \times T_{\chi}+S_{\chi}$ during $\left[t_{0}, t_{0}+T_{\chi}\right]$, and the driving distance $d_{\chi, 2}=v_{2} \times\left(\Delta T_{\chi+1}+T_{\chi+1}-T_{\chi}\right)$ during $\left[t_{0}+T_{\chi}, t_{0}+\Delta T_{\chi+1}+T_{\chi+1}\right]$. Therefore, the total driving distance $d_{\chi}=d_{\chi, 1}+d_{\chi, 2}=v_{2} \times\left(\Delta T_{\chi+1}+T_{\chi+1}\right)+S_{\chi}$ during $\left[t_{0}, t_{0}+\Delta T_{\chi+1}+T_{\chi+1}\right]$. (2) The driving distance $d_{\chi+1,1}=v_{1} \times \Delta T_{\chi+1}$ during $\left[t_{0}, t_{0}+\Delta T_{\chi+1}\right]$, and $d_{\chi+1,2}=v_{2} \times T_{\chi+1}+S_{\chi+1}$ during $\left[t_{0}+\Delta T_{\chi+1}, t_{0}+\Delta T_{\chi+1}+T_{\chi+1}\right]$. Therefore, the total driving distance $d_{\chi+1}=d_{\chi+1,1}+d_{\chi+1,2}=v_{1} \times \Delta T_{\chi+1}+v_{2} \times T_{\chi+1}+S_{\chi+1}$.

Because $d_{\chi+1}-d_{\chi}=L_{1}-L_{2}$, we can conclude that $\left(v_{1} \times \Delta T_{\chi+1}+v_{2} \times T_{\chi+1}+S_{\chi+1}\right)-$ $\left(v_{2} \times\left(\Delta T_{\chi+1}+T_{\chi+1}\right)+S_{\chi}\right)=L_{1}-L_{2}$. Therefore, $\quad S_{\chi+1}-S_{\chi}=\left(L_{1}-L_{2}\right)-\left(v_{1}-v_{2}\right) \times$ $\Delta T_{\chi+1}$, and the theorem is proved.

Theorem 2 If the reaction times of all the vehicles in an individual buffer are $\frac{L_{1}-L_{2}}{v_{1}-v_{2}}$, all the vehicles have the same acceleration strength.

Proof For two consecutive vehicles $\chi$ and $\chi+1$, if their reaction times are both $\frac{L_{1}-L_{2}}{v_{1}-v_{2}}$, then $S_{\chi}=S_{\chi+1}$ according to Theorem 1. $\Delta B T=\frac{L_{1}-L_{2}}{v_{1}-v_{2}}$ is called the balanced reaction time. 
Theorem 3 Inside the buffer, $\Delta t_{\chi}=\frac{\chi \times\left(L_{1}-L_{2}\right)-S_{\chi}}{v_{1}-v_{2}}$, where $\Delta t_{\chi}$ is the total time interval for vehicle $\chi$ to change velocity.

Proof Assume that the several behaviours shown in Fig. 3 are performed, and let $t_{0}$ be the initial time moment. For a vehicle $\chi$ during $\left[t_{0}, t_{0}+\Delta t_{\chi}+T_{\chi}\right]$, on one hand, the driving distance $d_{\chi}=v_{1} \times\left(t_{0}+\Delta t_{\chi}\right)+\left(S_{\chi}+v_{2} \times T_{\chi}\right)$; on the other hand, $d_{\chi}=v_{2} \times$ $\left(t_{0}+\Delta t_{\chi}+T_{\chi}\right)+\chi \times\left(L_{1}-L_{2}\right)$. Therefore, we can conclude that $\Delta t_{\chi}=\frac{\chi \times\left(L_{1}-L_{2}\right)-S_{\chi}}{v_{1}-v_{2}}$, and this proves the theorem.

Both before and after the acceleration (or deceleration) process in an individual buffer, the initial and terminal conditions are fixed and the states of two consecutive regions 1 and 2 are known. Moreover, according to Theorem 3, the starting time $\Delta t_{\chi}$ and acceleration strength $S_{\chi}$ have a one-to-one relationship. The length of the buffer $B$ is

$$
\frac{\rho_{2} \times\left(v_{2}-v_{1}\right) \times T_{\chi}}{\left(\rho_{2}-\rho_{1}\right)}
$$

where $T_{\chi}$ denotes the vehicle acceleration time, which is a flexible variable.

It must be mentioned that a special scenario arises when the vehicles change their velocities from $v_{1}$ to $v_{3}$ (rather than $v_{2}$ ) between regions $\operatorname{Re}_{1}\left(\rho_{1}, v_{1}, f_{1}\right)$ and $\operatorname{Re}_{2}\left(\rho_{2}, v_{2}, f_{2}\right)$. In this scenario, a new equilibrium state $\operatorname{Re}_{3}\left(\rho_{3}, v_{3}, f_{3}\right)$ is generated between $R e_{1}$ and $R e_{2}$. The two non-interactive buffers $B(1,3)$ and $B(3,2)$ segregate $R e_{1}, R e_{3}$, and $R e_{2} . B(1,3)$ and $B(3,2)$ are similar to $B(1,2)$. For example, when $R e_{1}$ is under a completely congested state and $R e_{2}$ is empty, the vehicles in $R e_{1}$ attempt to accelerate from $v_{1}=0$ to the free velocity $v_{2}$. However, a new state $\operatorname{Re}_{3}\left(\rho_{3}, v_{3}, f_{3}\right)$ arises between $\operatorname{Re}_{1}$ and $\operatorname{Re}_{2}$, where $v_{3} \approx v_{2}$ and $\rho_{3}>0$ because vehicle drivers do not maintain a very long distance from the vehicles in the front (this is consistent with common sense). $B(1,3)$ denotes the boundary of $R e_{1}$, and the position of $B(3,2)$ is where the first vehicle starts to accelerate. The vehicles accelerate from $v_{1}$ to $v_{2}$ in an early phase. However, in a later phase, the vehicles accelerate from $v_{1}$ to $v_{3}$, even if $v_{3}$ is very close to $v_{2}$, but $\rho_{3}>\rho_{2}$.

\section{Two meeting buffers}

Given three regions $\operatorname{Re}_{1}\left(\rho_{1}, v_{1}, f_{1}\right), \operatorname{Re}_{2}\left(\rho_{2}, v_{2}, f_{2}\right)$, and $\operatorname{Re}_{3}\left(\rho_{3}, v_{3}, f_{3}\right)$, the buffers $B(1,2)$ and $B(2,3)$ are located between the regions $R e_{1}$ and $R e_{2}$, and between $R e_{2}$ and $R e_{3}$, respectively. Let $\Delta B T_{1}, \Delta B T_{2}$, and $\Delta B T_{3}$ be the balanced reaction times in $B(1,2)$, $B(2,3)$, and $B(1,3)$, respectively. The terminal buffer $B(1,3)$ is a result of interaction between $B(1,2)$ and $B(2,3)$. In other words, the vehicles in buffer $B(1,3)$ change their velocities from $v_{1}$ to $v_{3}$, whereas those in the buffers $B(1,2)$ and $B(2,3)$ change their velocities from $v_{1}$ to $v_{2}$ and from $v_{2}$ to $v_{3}$, respectively.

Theorem 4 If buffer $B(1,2)$ catches up with buffer $B(2,3), \Delta B T_{1}>\Delta B T_{2}$, and $\Delta B T_{1}-\Delta B T_{3}=\frac{v_{2}-v_{3}}{v_{1}-v_{3}} \times\left(\Delta B T_{1}-\Delta B T_{2}\right)$.

Proof Let the downstream moving direction be positive. (1) The speed $v_{c 1}$ of buffer $B(1,2)$ is $\frac{\rho_{2} \times v_{2}-\rho_{1} \times v_{1}}{\rho_{2}-\rho_{1}}$, and the speed $v_{c 2}$ of buffer $B(2,3)$ is $\frac{\rho_{3} \times v_{3}-\rho_{2} \times v_{2}}{\rho_{3}-\rho_{2}} \cdot v_{c 1}>v_{c 2}$ if buffer $B(1,2)$ catches 
up with buffer $B(2,3) . \Delta B T_{1}=\frac{\frac{1}{\rho_{1}}-\frac{1}{\rho_{2}}}{v_{1}-v_{2}}=\frac{\frac{1}{\rho_{1}}}{v_{1}-v_{c 1}}=\frac{\frac{1}{\rho_{2}}}{v_{2}-v_{c 1}}$, and $\Delta B T_{2}=\frac{\frac{1}{\rho_{2}}-\frac{1}{\rho_{3}}}{v_{2}-v_{3}}=\frac{\frac{1}{\rho_{2}}}{v_{2}-v_{c 2}}=\frac{\frac{1}{\rho_{3}}}{v_{3}-v_{c 2}}$. Therefore, $\Delta B T_{1}>\Delta B T_{2}$ because $v_{c 1}>v_{c 2}$.

(2) $\Delta B T_{1} \times\left(v_{1}-v_{2}\right)=\frac{1}{\rho_{1}}-\frac{1}{\rho_{2}}, \Delta B T_{2} \times\left(v_{2}-v_{3}\right)=\frac{1}{\rho_{2}}-\frac{1}{\rho_{3}}$, and $\Delta B T_{3} \times\left(v_{1}-v_{3}\right)=$ $\frac{1}{\rho_{1}}-\frac{1}{\rho_{3}}$. Thus, $\Delta B T_{3} \times\left(v_{1}-v_{3}\right)=\Delta B T_{1} \times\left(v_{1}-v_{2}\right)+\Delta B T_{2} \times\left(v_{2}-v_{3}\right)$. Therefore, we can conclude that $\Delta B T_{1}-\Delta B T_{3}=\frac{\left(v_{2}-v_{3}\right)}{v_{1}-v_{3}} \times\left(\Delta B T_{1}-\Delta B T_{2}\right)$.

By combining (1) and (2), the theorem is proved.

When two buffers meet, the reaction times show systemic deviations. For example, an arbitrary vehicle in buffer $B(1,2)$ changes its velocity from $v_{1}$ to $v_{2}$, and the velocities in buffer $B(2,3)$ are changed from $v_{2}$ to $v_{3}$. Let $\Delta B T_{1}, \Delta B T_{2}$, and $\Delta B T_{3}$ be the balanced reaction times from $v_{1}$ to $v_{2}, v_{2}$ to $v_{3}$, and $v_{1}$ to $v_{3}$, respectively. Initially, the reaction times remain being $\Delta B T_{1}$ because there is no influence by the buffer $B(2,3)$ yet. After some time, the reaction times are gradually changed to $\Delta B T_{3}$. This process can be divided into three stages according to the different reaction times as follows.

Stage-1 Let the number of vehicles in Stage-1 be $m_{1}$. All the reaction times in this stage are approximately $\Delta B T_{1}$, and the acceleration strength is $S_{\chi}=S_{\chi-1}+\left(L_{1}-L_{3}\right)-$ $\left(v_{1}-v_{3}\right) \times \Delta T_{\chi}$, according to Theorem 1 . We can conclude that $S_{\chi}=S_{\chi-1}+\left(L_{1}-L_{3}\right)-$ $\left(v_{1}-v_{3}\right) \times \Delta B T_{1}=S_{1}+(\chi-1) \times\left[\left(L_{1}-L_{3}\right)-\left(v_{1}-v_{3}\right) \times \frac{L_{1}-L_{2}}{v_{1}-v_{2}}\right]$, where $1 \leqslant \chi \leqslant m_{1}$.

Stage-2 In Stage-2, the reaction time is changed from $\Delta B T_{1}$ to $\Delta B T_{3}$, where $\Delta B T_{1} \neq \Delta B T_{3}$ and $\Delta B T_{1}-\Delta B T_{3}=\frac{v_{2}-v_{3}}{v_{1}-v_{3}} \times\left(\Delta B T_{1}-\Delta B T_{2}\right)$, according to Theorem 3. The velocities in this stage are limited to $\left[0, v_{f}\right]$. If the velocities are changed to zero or $v_{f}$, Stage- 2 terminates and Stage- 3 begins.

Let the number of vehicles in Stage-2 be $m_{2} . S_{\chi}=S_{\chi-1}+\left(L_{1}-L_{3}\right)-\left(v_{1}-v_{3}\right) \times$ $\Delta T_{\chi}=S_{\chi-1}+\left(v_{1}-v_{3}\right) \times\left(\Delta B T_{3}-\Delta T_{\chi}\right)$, where $m_{1}<\chi \leq m_{1}+m_{2}$. For any vehicle $\chi$ in Stage-2, the time when it starts changing its velocity is $m_{1} \times \Delta B T_{1}+\Sigma_{k=m_{1}+1}^{\chi}\left[\Delta B T_{3}-\right.$ $\left.\left(\Delta B T_{3}-\Delta T_{k}\right)\right]$, and its acceleration strength is $S_{\chi}=S_{m_{1}}+\left(v_{1}-v_{3}\right) \sum_{k=m_{1}+1}^{\chi}\left(\Delta B T_{3}-\Delta T_{k}\right)$.

From the statements above, for any vehicle $1 \leq \chi \leq m_{1}+m_{2}$, the time when it starts changing its velocity is $\Sigma_{k=1}^{\chi}\left(\Delta B T_{3}-\Delta \Delta T_{k}\right)$, and its acceleration strength is $S_{\chi}=S_{1}+\left(v_{1}-v_{3}\right)$ $\Sigma_{k=1}^{\chi}\left(\Delta B T_{3}-\Delta T_{k}\right)=S_{1}+\left(v_{1}-v_{3}\right) \Sigma_{k=1}^{\chi}\left(\Delta T_{k}\right)$, where $\Delta \Delta T_{k}=\Delta B T_{3}-\Delta T_{k}$. If $1 \leq \chi \leq m_{1}$, $\Delta \Delta T_{\chi}=\Delta B T_{3}-\Delta B T_{1}$; otherwise, $\Delta \Delta T_{k}$ varies between $\Delta B T_{1}$ and $\Delta B T_{3}$.

Stage-3 In this stage, the vehicles change their velocities from $v_{1}$ to 0 or $v_{f}$ and then to $v_{3}$. Considering Fig. $4 \mathrm{a}$ as an example, in some scenarios, velocities of the vehicles continuously decrease towards 0 , even if their velocities have reached $v_{3}$, because a safe distance must be maintained for any neighbouring vehicles. In this case, a new and completely stopped queue $Q_{m}$ occurs in the interior of this buffer. The merged buffer is divided into two independent buffers by $Q_{m}$. The length of $Q_{m}$ depends on the reaction times of the vehicles in the front and rear of $Q_{m}$.

From the statements above, it is concluded that the temporal evolution of buffers depends on the difference in traffic states. As for the spatial evolution, the buffer length 


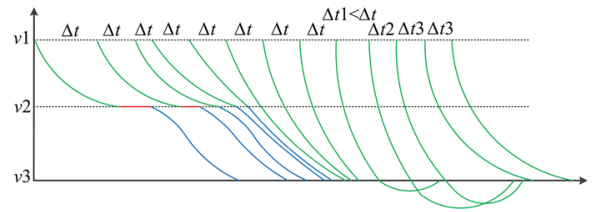

(a)

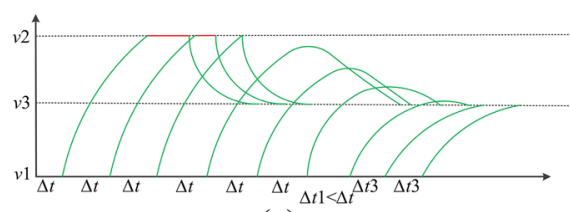

(c)

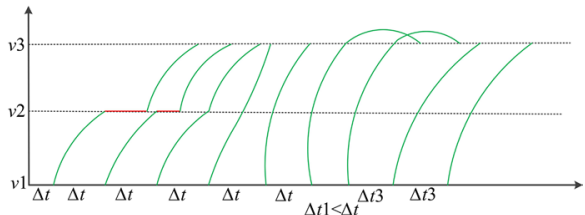

(b)

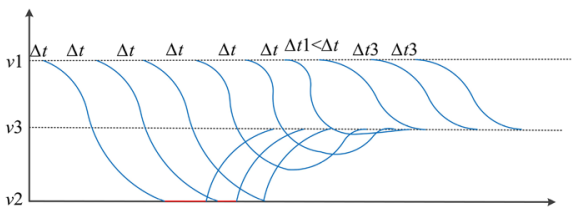

(d)

Fig. 4 Varying vehicle behaviors in two meeting buffers, where solid lines denote the dynamic velocity, the values on the horizontal axis are the time interval for a vehicle starts to change velocity, and the vertical axis is about the vehicles' velocities

remains constant (i.e., $\frac{\rho_{2} \times\left(v_{2}-v_{1}\right) \times T_{X}}{\left(\rho_{2}-\rho_{1}\right)}$ ) in a single shockwave; while in two interactive shockwaves, the buffer lengths range from $\left[\frac{\rho_{2} \times\left(v_{2}-v_{1}\right)}{\rho_{2}-\rho_{1}}+\frac{\rho_{3} \times\left(v_{3}-v_{2}\right)}{\rho_{3}-\rho_{2}}\right] \times T_{\chi}$ to $\frac{\rho_{3} \times\left(v_{3}-v_{1}\right) \times T_{X}}{\rho_{3}-\rho_{1}}$, and finally evolve into one or two independent buffers, as was introduced in Stage-3.

The velocities in the conventional LWR model are immediately changed without considering the non-equilibrium states. The RLWR model discusses the drivers' microscopic behaviours during the acceleration (or deceleration) process, which allows the velocities to be around equilibrium values and avoids negative flows and velocities. The RLWR model overcomes the drawbacks of the conventional LWR model and the limitations of Payne-type models to a certain extent.

\section{RSM on traffic}

When using the RSM to describe dynamic traffic flows, ripples refer to shockwaves where the traffic states at the upstream and downstream directions are different; water and stakes represent roads and intersections in a transportation network, respectively. The stones represent the disturbances caused by the dynamic OD matrix, disruptions, and dynamic drivers' route choices. The dynamic traffic shockwaves correspond to the spreading ripples in the RSM.

\section{RLWR-RSM}

The RLWR model is integrated with the RSM to construct a hybrid RLWR-RSM. The three main processes discussed below are embedded when the RLWR-RSM is used to model dynamic traffic flows. 


\section{Shockwave generation}

If no disturbance exists, the transportation network is a stable system. On an arbitrary single road $(i, j)$, the flow rate $q_{i j}$, density $\rho_{i j}$, and velocity $v_{i j}$ are constant. However, disturbances cause instability in the traffic system. For example, when rush hours approach, the time-varying OD matrix $M_{O D}(t)$ increases the traffic flows and causes congestion in a transportation network; however, the congestion is terminated at later periods.

(1) Time-varying OD matrix Let $M_{O D}(t)$ be the time-varying OD matrix and $S_{i j}(t) \in M_{O D}(t)$ be the vehicle flow rate from node $i$ to $j$ at time moment $t$, as introduced in Sect. 2. Let $(i, u)$ be an outgoing road of node $i, P_{i, j}^{k}(1 \leqslant k \leqslant w)$ be a route choice to $j$, and $A_{i j}^{k} \in S_{i j}(t)$ be the percentage of vehicles whose path choices are $P_{i, j}^{k}$. Furthermore, the inflow $S_{i u}^{i j}(t)$ from $S_{i j}(t)$ to road $(i, u)$ is $\sum_{k=1}^{w}\left(A_{i j}^{k} \times S_{i j}(t) \times \alpha\right)$, where, $\alpha=1$ if $u \in P_{i, j}^{k}$, and $\alpha=0$ otherwise. Similarly, the total inflow $S_{i u}^{O D}$ from the time-varying OD matrix to road $(i, u)$ is $\sum_{j=1}^{n} S_{i u}^{i j}(t)$.

The inflows on road $(i, u)$ are not only from the OD matrix but also from incoming roads. Let road $(v, i)$ be an incoming road of $(i, u)$ and $O_{v i}=\left\{\left(F_{1}^{\text {out }}, D_{1}^{\text {out }}, P_{1}^{\text {out }}\right), \cdots\right.$, $\left.\left(F_{w}^{\text {out }}, D_{w}^{\text {out }}, P_{w}^{\text {out }}\right)\right\}$. Then, the flows $F_{i u}^{v i}$ from $(v, i)$ to $(i, u)$ are $\sum_{k=1}^{w}\left(F_{k}^{\text {out }} \times \alpha\right)$, where $\alpha=1$ if $u \in P_{k}^{\text {out }}$, and $\alpha=0$ otherwise. Therefore, the total inflow $S_{i u}^{U p}$ of $(i, u)$ from all incoming roads is $\sum_{k=1}^{n}\left(F_{i u}^{k i} \times \beta\right)$, where $\beta=1$ if $(k, i) \in A$, and $\beta=0$ otherwise.

Therefore, the total inflow $F_{i u}^{i n}(t)=S_{i u}^{O D}+S_{i u}^{U p}$ at the time moment $t$ on road $(i, u)$ can be determined. From time moment $t-1$ to $t$, if either the flow value of $F_{i u}^{i n}(t)$ or the information $\left(F_{k}^{i n}, D_{k}^{i n}, P_{k}^{i n}\right) \in F_{i u}^{i n}(t)$ changes, a new shockwave $R_{h}$ is generated. As shown in Fig. 5, $R_{h}\left(I_{u}\right)=F_{i u}^{i n}(t)$ and $R_{h}(v)=\frac{q_{h}-q_{h-1}}{\rho_{h}-\rho_{h-1}} . R_{h}$ moves upstream if $R_{h}(v)<0$ or downstream if $R_{h}(v)>0$; otherwise, the position is retained.

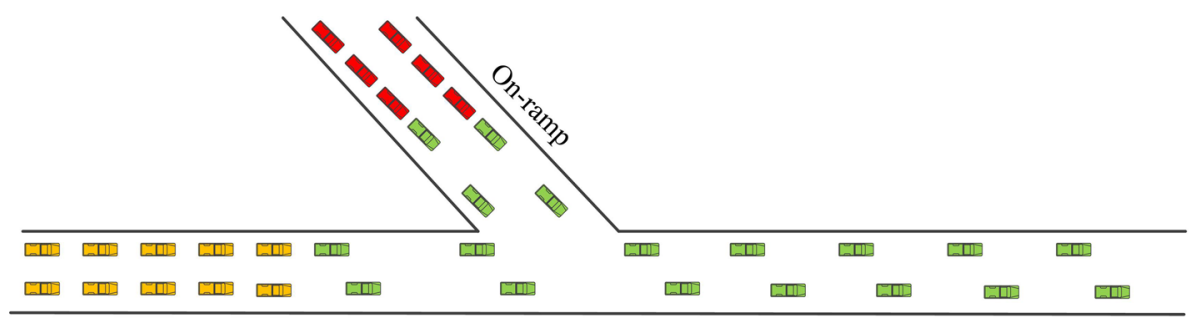

(a)

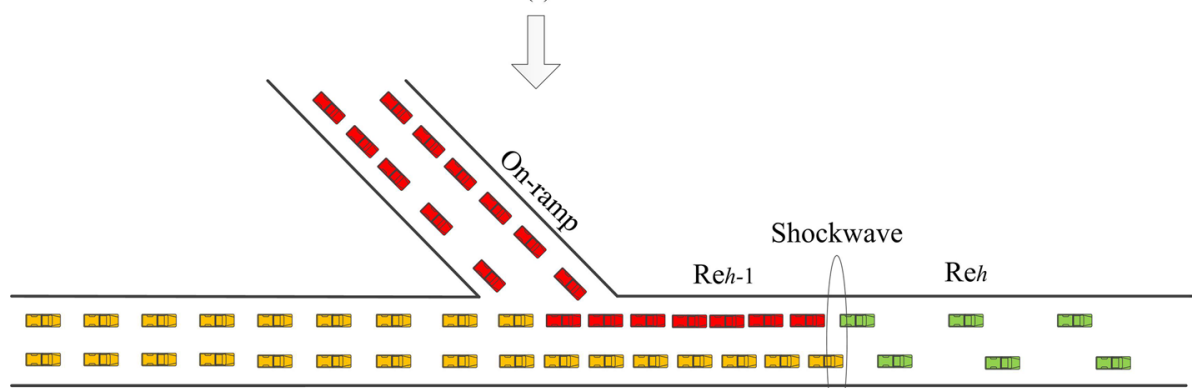

(b)

Fig. 5 The mechanism of generating shockwaves due to time-varying OD matrix 
The number of shockwaves in a network depends on the sampling period of the time-varying OD matrix, for which two extreme conditions are considered. If the sampling period of the OD matrix is long, very few shockwaves are available to accurately describe the dynamic traffic states. If the OD matrix changes frequently, the RLWR-RSM is similar to micro models that involve a high computational complexity. Time complexity and accuracy are in conflict. Experiments conducted in later sections demonstrate the modelled traffic states with different sampling periods.

(2) Dynamic route choices The previous section had already described how route choices affect the flow distribution (Fig. 6). Generally, route choices are classified as static or dynamic choices. Drivers tend to make static choices in free roads where the vehicles can travel at the maximum velocity, and static routes are always the same as dynamic routes in this case; dynamic route choices would be better under congested traffic conditions because they avoid congested roads (Sever et al. 2013). Let $S P$ and $D P$ be the percentages of vehicles that follow the static and dynamic route choices, respectively, where $S P+D P=100 \%$.

(3) Dynamic network disruption Three shockwaves described how route choices affect the flow distribution $R_{1}, R_{2}$, and $R_{3}$ are triggered when a disruption $\operatorname{Drp}\left(D_{p}, T_{p}, C_{d}, R_{c}\right)$ occurs (as shown in Fig. 7). Let $q_{0}, \rho_{0}$, and $I_{0}$ be the vehicle flow rate, density, and vehicle information, respectively, in location $D_{p}$ before disruption. The new flow rate after disruption is $q_{1}=C_{d}$. Let $\rho_{1} \geq \rho_{B}$ and $\rho_{2} \leq \rho_{B}$ be the new densities whose corresponding flow rates are both $q_{1}$. Shockwave $R_{1}$ has an inflow $q_{0}$, outflow $q_{1}$, upstream energy $R_{1}\left(E_{u}\right)=\rho_{0}$, downstream energy $R_{1}\left(E_{d}\right)=\rho_{1}, R_{1}(v)=\frac{q_{1}-q_{0}}{\rho_{1}-\rho_{0}}<0$, and $R_{1}\left(I_{u}\right)=I_{0}$. Shockwave $R_{2}$ has an

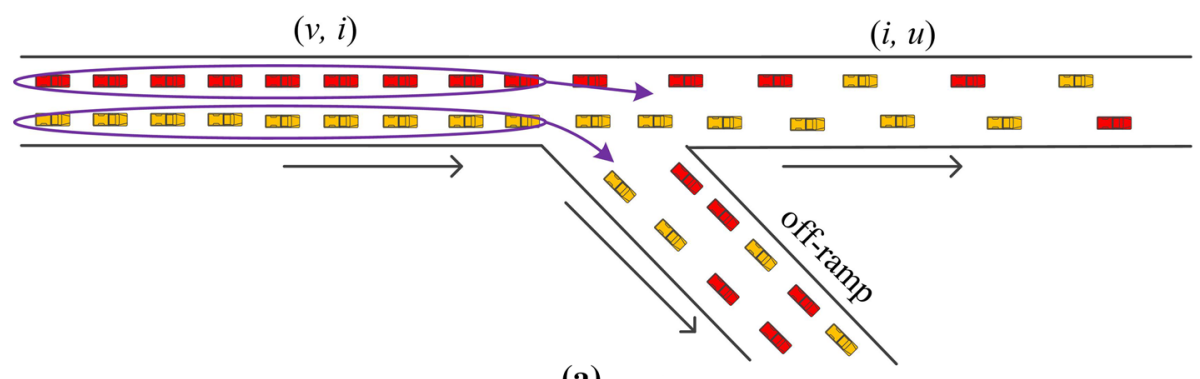

(a)

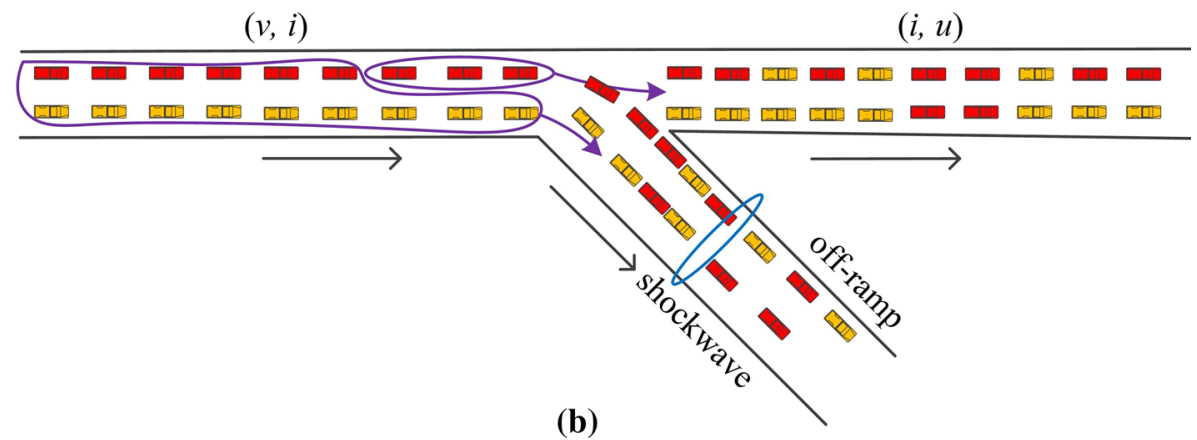

Fig. 6 The mechanism of generating shockwaves due to dynamic route choices 
$\left(v_{0}, \rho_{0}, q_{0}\right)$

\begin{tabular}{|c|c|c|c|c|c|c|}
\hline पID & पII & 미 & $\square \square$ & 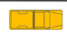 & पII & 口II \\
\hline 口[1] & 口II & पIII) & $\square$ & पII & $\square$ & 口II \\
\hline
\end{tabular}

(a)

Disruption

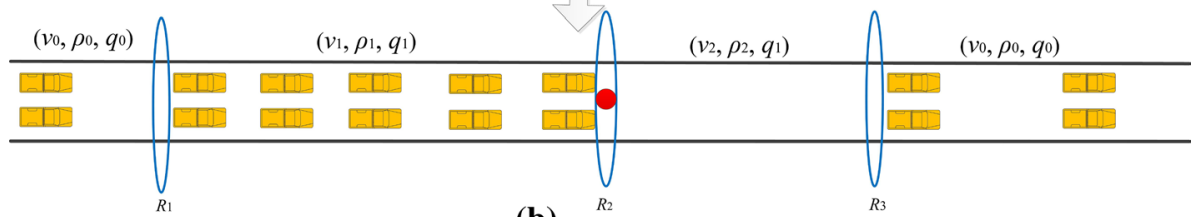

(b)

Fig. 7 The mechanism of generating shockwaves due to disruption

inflow $q_{1}$, outflow $q_{1}$, upstream energy $R_{2}\left(E_{u}\right)=\rho_{1}$, downstream energy $R_{2}\left(E_{d}\right)=\rho_{2}$, $R_{2}(v)=\frac{q_{1}-q_{1}}{\rho_{2}-\rho_{1}}=0$, and $R_{2}\left(I_{u}\right)=\frac{q_{1}}{q_{0}} \times I_{0}$. Shockwave $R_{3}$ has an inflow $q_{1}$, outflow $q_{0}$, upstream energy $R_{3}\left(E_{u}\right)=\rho_{2}$, downstream energy $R_{3}\left(E_{d}\right)=\rho_{0}, R_{3}(v)=\frac{q_{0}-q_{1}}{\rho_{0}-\rho_{2}}>0$, and $R_{3}\left(I_{u}\right)=\frac{q_{1}}{q_{0}} \times I_{0}$.

Disruption is a dynamic process which gradually recovers until the densities at the two sides are the same. $R_{c}$ is the recovery rate of capacity. The new inflow and outflow of $R_{2}$ at the time moment $t>T_{p}$ are $q_{3}=\min \left(q_{0}+R_{c} \times\left(t-T_{p}\right), u_{i j}\right)$. Let $\rho_{3} \geqslant \rho_{B}$ and $\rho_{4} \leqslant \rho_{B}$ be the new densities whose corresponding flows are both $q_{3}$. Then, the inflow, outflow, upstream energy, and downstream energy of $R_{2}$ become $q_{3}, q_{3}, \rho_{3}$, and $\rho_{4}$, respectively. $R_{2}(v)=\frac{q_{3}-q_{3}}{\rho_{4}-\rho_{3}}=0$ and $R_{2}\left(I_{u}\right)=\frac{q_{3}}{q_{1}} \times R_{2}\left(I_{u}\right)$. A new shockwave $R_{4}$ is generated in the upstream of $D_{p} . R_{4}$ has an inflow $q_{1}$, outflow $q_{3}$, upstream energy $\rho_{1}$, downstream energy $\rho_{3}$, $R_{4}(v)=\frac{q_{3}-q_{1}}{\rho_{3}-\rho_{1}}<0$, and $R_{4}\left(I_{u}\right)=R_{2}\left(I_{u}\right)$. Another new shockwave $R_{5}$ is generated in the downstream of $D_{p}$. $R_{5}$ has an inflow $q_{3}$, outflow $q_{1}$, upstream energy $\rho_{4}$, downstream energy $\rho_{2}, R_{5}(v)=\frac{q_{1}-q_{3}}{\rho_{2}-\rho_{4}}>0$, and $R_{5}\left(I_{u}\right)=R_{2}\left(I_{u}\right)$. Based on this, let $q_{1}=q_{3}, \rho_{3}=\rho_{1}$, and $\rho_{2}=\rho_{4}$; then, similar shockwaves as $R_{4}$ and $R_{5}$ are continuously generated until the densities at the upstream and downstream directions of $R_{2}$ become the same.

\section{Shockwave movement}

Let $\left(\rho_{1}, v_{1}, q_{1}\right)$ and $\left(\rho_{2}, v_{2}, q_{2}\right)$ be the traffic states at the upstream and downstream directions of the shockwave $R$, respectively. The shockwave speed is $-\infty<R(v)=\frac{q_{2}-q_{1}}{\rho_{2}-\rho_{1}} \leqslant \min \left(v_{1}, v_{2}\right) . R(v)<0$ approximately implies that the traffic states downstream profoundly influence the entire road. Vehicles continue moving downstream regardless of the shockwave speed being negative or positive. After the generation of a new shockwave $R$, a corresponding auxiliary shockwave $R_{a}$ is triggered at the downstream direction of $R$ because the vehicles velocities are higher than the shockwave speed. $R_{a}$ has the same densities $\rho_{2}$ and velocities $v_{2}$, but different vehicle information as $R$ for the two directions. The upstream vehicle information is $R_{a}\left(I_{u}\right)=R\left(I_{u}\right) \times \frac{q_{2}}{q_{1}}$, and the downstream vehicle information is $R_{a}\left(I_{d}\right)=R\left(I_{d}\right)$. The auxiliary shockwave does not influence the vehicle velocity, but different vehicle information (the vehicles have different route choices and destinations) causes different vehicle flow distributions. Differing from the conventional RSM, there is no loss of energy during shockwave movement. 


\section{Shockwave interaction}

Consequent shockwaves $R_{1}$ and $R_{2}$ with different speeds may encounter each other on the same road and may be replaced by a new merged shockwave $R_{m} . R_{m}\left(I_{u}\right)=R_{1}\left(I_{u}\right)$, $R_{m}\left(I_{d}\right)=R_{2}\left(I_{d}\right)$, and $R_{m}(v)=\frac{q_{3}-q_{1}}{\rho_{3}-\rho_{1}}$. The buffer of $R_{m}$ is discussed in Sect. 4.1.

When a shockwave $R$ reaches a node, new shockwaves are generated in the outgoing or incoming roads. Let us assume that $R$ reaches the exit of $\operatorname{road}(i, j) \in A$; the next operation involves the traffic flow redistribution collaborating with the time-varying OD matrix and dynamic route choices. According to the methods introduced for the 'dynamic OD matrix' (Sect. 4.3), a new shockwave is triggered at the entry of the outgoing road $(j, v)$. The characteristics of each vehicle's route choice and flow conservation principle are maintained.

If the shockwave $R$ reaches the entry of road $(i, j)$, the reverse operation of flow redistribution is performed for the incoming roads. The shockwave is actually the changed traffic flows. The arrival of a congestion-recovery shockwave indicates the elimination of congestion and allows more flows to the road. Following the flow redistribution method, the congestion-recovery shockwave spreads to the incoming roads if they are under congested traffic conditions. Let $(u, i)$ be an incoming road of node $i$ with density $\rho_{u, i}>\rho_{B}$, let vehicle information at the exit of $(u, i)$ be $\left\{\left(F_{1}, D_{1}, P_{1}\right), \cdots,\left(F_{w}, D_{w}, P_{w}\right)\right\}$, and let $F_{i, j}^{u, i}=\sum_{k=1}^{w}\left(F_{k} \times \gamma\right)\left(\gamma=1\right.$ if $j \in P_{k}$, and $\gamma=0$ otherwise). Then, a new shockwave $R_{\text {new }}$ is triggered at the exit of $(u, i)$ and its parameters are set as follows: 1) $R_{\text {new }}\left(I_{u}\right)=\left\{\left(F_{1}, D_{1}, P_{1}\right), \cdots,\left(F_{w}, D_{w}, P_{w}\right)\right\}$ and $R_{\text {new }}\left(I_{d}\right)=\sum_{k=1}^{w}\left(F_{k} \times \lambda, D_{k}, P_{k}\right)$, where $\lambda=\frac{F_{2}}{F_{1}}>1$ if $j \in P_{k}, \lambda=1$ otherwise. 2) Upstream density $\rho_{3}=\rho_{u, i}$ and downstream density $\rho_{4}<\rho_{B}$ whose corresponding flows are $\left|R_{\text {new }}\left(I_{d}\right)\right|$. 3) Shockwave speed $R_{\text {new }}(v)=\frac{\left|R_{\text {new }}\left(I_{d}\right)\right|-\left|R_{\text {new }}\left(I_{u}\right)\right|}{\rho_{4}-\rho_{3}}<0$. In addition to incoming roads, vehicle flow rate $S_{i k}(t)$ in the OD matrix is also affected. If the actual vehicle flow rate $S_{i k}^{a}(t)$ is less than the expected rate $S_{i k}(t)$, it is permissible to increase the actual inputs to the transportation network. $\sum_{k=1}^{n} S_{i, k}(t)$ is treated as the flow onto a virtual incoming road of $i$, and the new actual inputs can be calculated similarly to other real incoming roads. However, the OD matrix and incoming roads can be affected by the congestion shockwave at the entry of $(i, j)$; in the congestionrecovery case, the spillback moves to incoming roads only when the OD matrix or incoming roads has/have surplus flow. The traffic flow redistribution follows the same formulas as in the congestion case, but $\lambda<1$.

The reversed operations of flow redistribution cannot guarantee that the vehicle information of the inflows is identical to that before redistribution. For instance, when a congestion-recovery shockwave reaches node $i$ and all incoming roads are congested, the inflows of $(i, j)$ are out of balance. The flow distribution operation should be re-performed, and corresponding auxiliary shockwaves are generated, even if the inflows remain constant, because vehicle information changes. If the inflows change, a new shockwave $R_{3}$ is triggered at the entry of $(i, j)$. It must be mentioned that $R_{3}$ cannot move upstream.

\section{Travel time estimation}

It is assumed that $m$ existing shockwaves $R_{i j-1}\left(t_{0}\right), R_{i j-2}\left(t_{0}\right), \cdots, R_{i j-m}\left(t_{0}\right)$ propagate in an individual road $(i, j)$ at the time moment $t_{0}$. The existing shockwaves continue to propagate and interact with each other, which can be simulated by the above methods. Besides, the new shockwaves $R_{d}(t)$ and $R_{u}(t)$ generated at the exit and entry of a road at 
time $t>t_{0}$ can be predicted according to Sect. 4.2. For a vehicle $\chi$ that enters the road $(i, j)$ at time $t_{0}$, the calculation procedure of the time taken to pass through $(i, j)$ is shown in Fig. 8.

The new shockwave $R_{u}(t)$ has no effect on the travel time of a vehicle $\chi$ if it is generated at the road entry after $t_{0}$ because the velocity $v$ of $\chi$ is determined only by the traffic conditions in front of it. As seen from Fig. 8, two important steps are executed to estimate the travel time: (1) calculating the minimum evolution time interval $t_{\min }$ and (2) changing the shockwaves' and vehicle $\chi$ 's states on the road.

Let $t$ be the current time moment, $t_{\min }=\min \left(t_{\min 1}, t_{\min 2}, t_{\min 3}, t_{\min 4}\right)$, where $t+t_{\min 1}$ is the earliest time moment when two shockwaves interact; $t+t_{\min 2}$ is the earliest time moment when a new shockwave is generated at (or exits) the exit of the road or a disruption is triggered in downstream; $t+t_{\min 3}$ is the earliest time moment when vehicle $\chi$ encounters an existing shockwave; and $t_{\min 4}$ is the consumed time for $\chi$ to reach the exit of the road if it travels at a constant velocity. During the time interval $\left(t, t+t_{\min }\right)$, the shockwaves do not interact, no new shockwave propagates out of (or onto) the exit of the road or generated in downstream, and the vehicle $\chi$ maintains a constant velocity and does not encounter any shockwave.

At the time moment $t+t_{\text {min }}$, two meeting shockwaves are merged if $t_{\min }=t_{\min 1}$. A new shockwave is generated in downstream or enters (or is kicked out of) the exit of the road if $t_{\min }=t_{\min 2} \cdot \chi$ changes its velocity if $t_{\min }=t_{\min 3}$; otherwise, $t_{\min }=t_{\min 4}$ and vehicle $\chi$ reaches the exit of the road which is the termination condition. To improve the accuracy of the evaluated travel times, the effects of buffer are further considered.

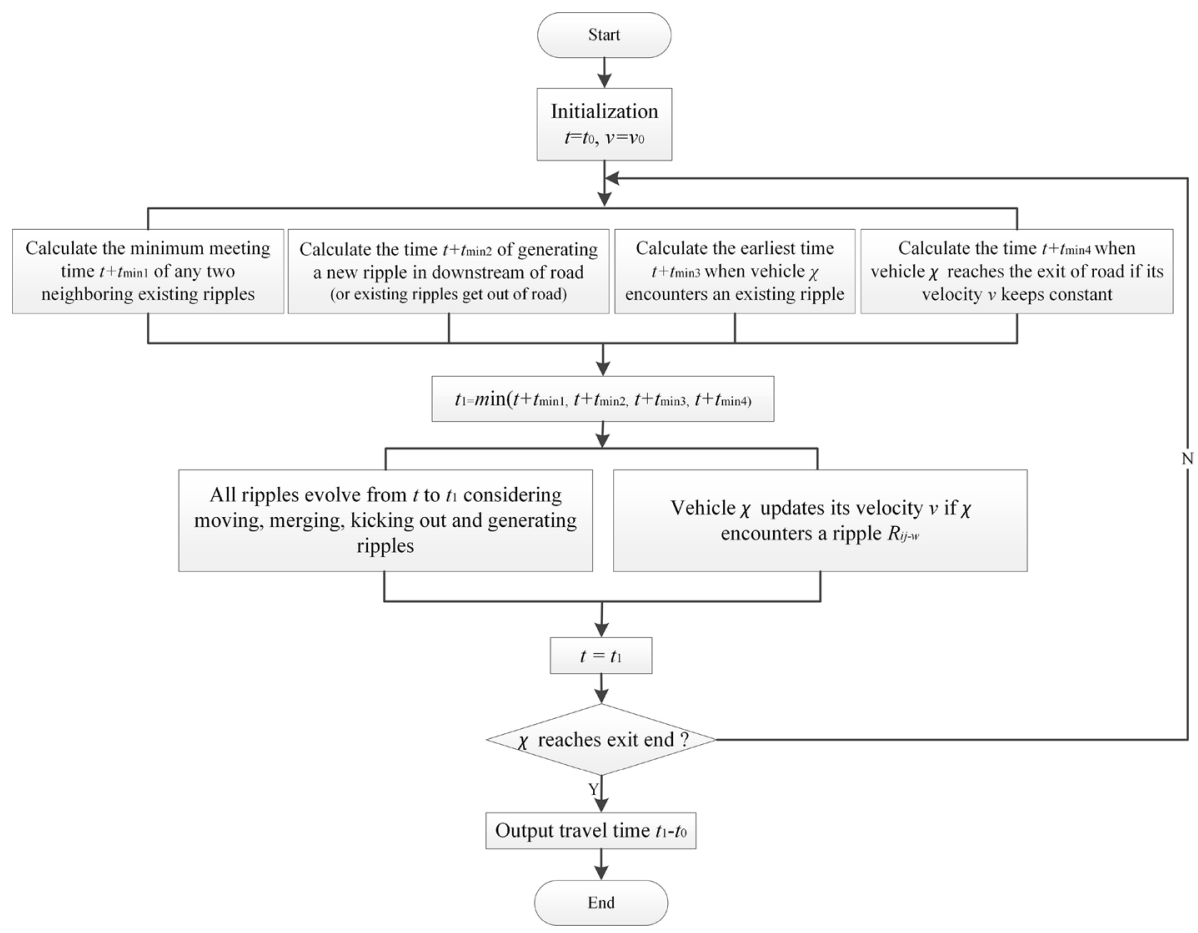

Fig. 8 The flow chart of evaluating travel times considering evolutive traffic conditions 
Let $\Delta T_{0}$ and $\Delta T_{1}$ be the starting times for $\chi$ to change velocity in the conventional LWR and RLWR models, respectively. When $\chi$ crosses a buffer $B(1,2)$ at the time moment $t$, let $S_{0}$ and $S_{1}$ be the acceleration strengths in the conventional LWR and RLWR models, respectively. $\Delta T_{0}=\Delta T_{1}$ in an individual buffer because all the vehicles have an approximate balanced reaction time $\Delta B T . S_{0}=0$ because the acceleration (or deceleration) in the conventional LWR model is instantaneous. $S_{1}$ is a variable determined by $v_{1}$ and $v_{2}$. Compared with the conventional LWR model, the travel time $T$ in the RLWR model is increased by a constant value $\frac{S_{1}}{v_{2}}$.

If $\chi$ goes through a merged buffer $B(1,3)$ as shown in Sect. 4.1 , it experiences one stage of $B(1,3)$. Let $\Delta B T_{1}, \Delta B T_{2}$, and $\Delta B T_{3}$ be the balanced reaction times of $B(1,2), B(2,3)$, and $B(1,3)$, respectively. Before determining its behaviour, the stage that will be experienced by the vehicle $\chi$ should be known. Let $m_{1}, m_{2}$, and $m_{3}$ be the number of vehicles that experience the first, second, and third stages, respectively.

(1) According to Theorem 3, $\Delta B T_{1}-\Delta B T_{3}=\frac{v_{2}-v_{3}}{v_{1}-v_{3}} \times\left(\Delta B T_{1}-\Delta B T_{2}\right)$, and $\Delta B T_{1}>\Delta B T_{2}$ when the buffer $B(1,2)$ catches up with the buffer $B(2,3)$. Let $m_{1}=\alpha \times \frac{|B(2,3)|}{R_{1}(v)-R_{2}(v)} \times$ $R_{1}(v) \times R_{2}\left(E_{u}\right)$, where $\alpha$ is a coefficient and $|B(2,3)|$ is the length of the buffer $B(2,3)$. Compared with the conventional LWR model, the travel times of vehicles in the first stage are increased by a constant value $\frac{S}{v_{2}}$.

(2) As expected, acceleration strengths in Stage-2 are changed from $S_{m 1}$ to $S_{m 1+m 2}$, where $S_{m 1}$ can be calculated in Stage- 1 and works as the initial condition. $S_{m 1+m 2}$ is the known termination condition. We assume that the difference of acceleration strengths between two neighbouring vehicles is a fixed value $\beta \times \frac{S_{m 1+m 2}-S_{m 1}}{\left|S_{m 1+m 2}-S_{m 1}\right|}$, where coefficient $\beta>0$. Therefore, the number $m_{2}$ of vehicles in Stage- 2 is $\frac{\left|S_{m 1+m 2}-S_{m 1}\right|}{\beta}$.

For the $k$ th vehicle in Stage-2, its starting time at which the velocity changes is $t_{k}=t_{0}+\left(m_{1}-1\right) \times \Delta B T_{1}+\sum_{m 1+1}^{k} \Delta T_{k}$, and its acceleration strength is $S_{k}=S_{m 1}+$ $\left(v_{1}-v_{3}\right) \times \sum_{m 1+1}^{k}\left(\Delta B T_{3}-\Delta T_{k}\right)$. Before $t_{k}$, its velocity is $v_{1}$. During $\left\lfloor t_{k}, t_{k}+T_{k}\right\rfloor$, its travel distance is $v_{3} \times T_{k}+S_{k}$. After $t_{k}+T_{k}$, its velocity is $v_{3}$. Its starting time to accelerate (or decelerate) is changed by $\Delta t=t_{1}-t_{0}-(k-1) \times \Delta B T_{1}=\left(m_{1}-k\right) \times \Delta B T_{1}+$ $\sum_{m 1+1}^{k} \Delta T_{k}$. Therefore, the travel times are decreased by $\left(v_{2}-v_{3}\right) \times \Delta t+\frac{S_{k}}{v_{3}}$.

(3) In place of region $R e_{2}$, a new region $\operatorname{Re}_{4}\left(\rho_{4}, v_{4}, f_{4}\right)$ arises between regions $R e_{1}$ and $R e_{3}$ in Stage-3, where $R e_{4}$ is either totally congested or free. Any vehicle $\chi$ in Stage-3 experiences two new independent buffers $B(1,4)$ and $B(4,3)$ and one new region $R_{4}$. Therefore, the travel times of $\chi$ can be calculated using the method in Stage-1.

Therefore, we can conclude that reaction times and acceleration strengths of vehicles when crossing a buffer/buffers can be used for an accurate evaluation of travel times. In an individual buffer, nearly stable vehicle behaviours are seen; in two or more interactive buffers, the chaotic process of merging buffers is known using detailed descriptions of vehicles' microscopic behaviours. In actuality, vehicle behaviours can not be accurately predicted because of the different reactivities and driving skills of drivers. Besides, driverless vehicles are an important development. A significant difference between human-driven and driverless vehicles is that driverless vehicles travel in a more predictable manner and consider the minimum safe distance between neighbouring vehicles and the comfort of passengers. Compared with human-driven vehicles, the RLWR-RSM model shows a relatively higher prediction accuracy for driverless vehicles, because it does not require a deep knowledge to describe the fluctuating traffic states caused by different drivers. 


\section{Numerical experiments and analysis}

To validate the effectiveness of the RLWR-RSM and the accuracy of estimated travel times, experiments were designed with varying inputs as follows.

\section{Experimental design}

(1) Network and fundamental diagram The artificial network has 4 intersections and 5 roads. The road capacities and lengths are shown in Fig. 9. The fundamental diagram for the roads in this network is shown in Table 1 and Fig. 10, where velocity and density have a one-to-one relationship, and the flow-density functions between two arbitrary contiguous samples are linear.

(2) Time-varying OD matrix To simulate the traffic conditions during peak hours, the number of vehicles that travel between any pair of origin and destination was initially increased and then decreased, as shown in "Appendix 1 and 2". The main difference between "Appendix 1 and 2" is the sampling period, which aims at testing the flexibility of the RLWR-RSM towards rapidly changing traffic conditions. "Appendix 3" is a static OD matrix which is used to test the influence of dynamic disruptions and route choices independently.

(3) Dynamic route choices Three available routes $P_{1}, P_{2}$, and $P_{3}$ ("Appendix 4") are used in this experiment. The route choices are shown in "Appendix 5", where the percentages of vehicles along $P_{2}$ and $P_{3}$ are the same, and the percentages of vehicles along $P_{1}$ in roads $(0,1),(0,3)$, and $(1,2)$ initially increase and then decrease, and they continuously decrease in roads $(0,2),(1,3)$, and $(3,2)$.

(4) Dynamic disruption To model the dynamic occurrence and recovery processes of disruption (such as a traffic accident), disruptions $\operatorname{Drp}_{1,2}(2000,0.3,150)$ and $\operatorname{Drp}_{1,2}(4000,0.4,150)$ are set on $\operatorname{road}(1,2)$, and a disruption $\operatorname{Drp}_{1,3}(1600,0.3,150)$ is set on $\operatorname{road}(1,3)$.

(5) Comparison with the CTM In order to confirm the reliability of the RLWR-RSM, two comparisons with the CTM are conducted on a highway with one on-ramp. The length of the road is $70 \mathrm{~km}$ and the on-ramp is $60 \mathrm{~km}$ away from the entry of the road. The cell

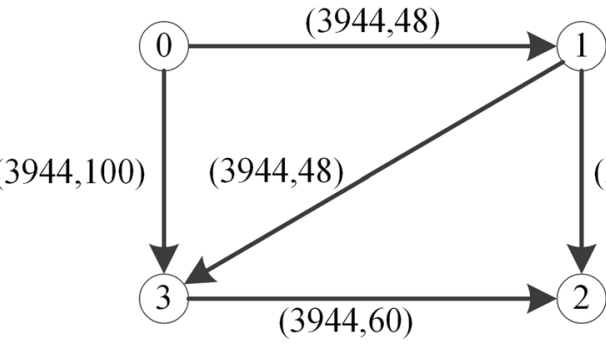

$(3944,100)$

$$
\begin{aligned}
& \text { Legend: } \\
& \begin{array}{l}
(i) \text { : Intersection } \\
\left(u_{i j}, l_{i j}\right): \text { (Capacity, length) }
\end{array}
\end{aligned}
$$

Fig. 9 The illustrative network 


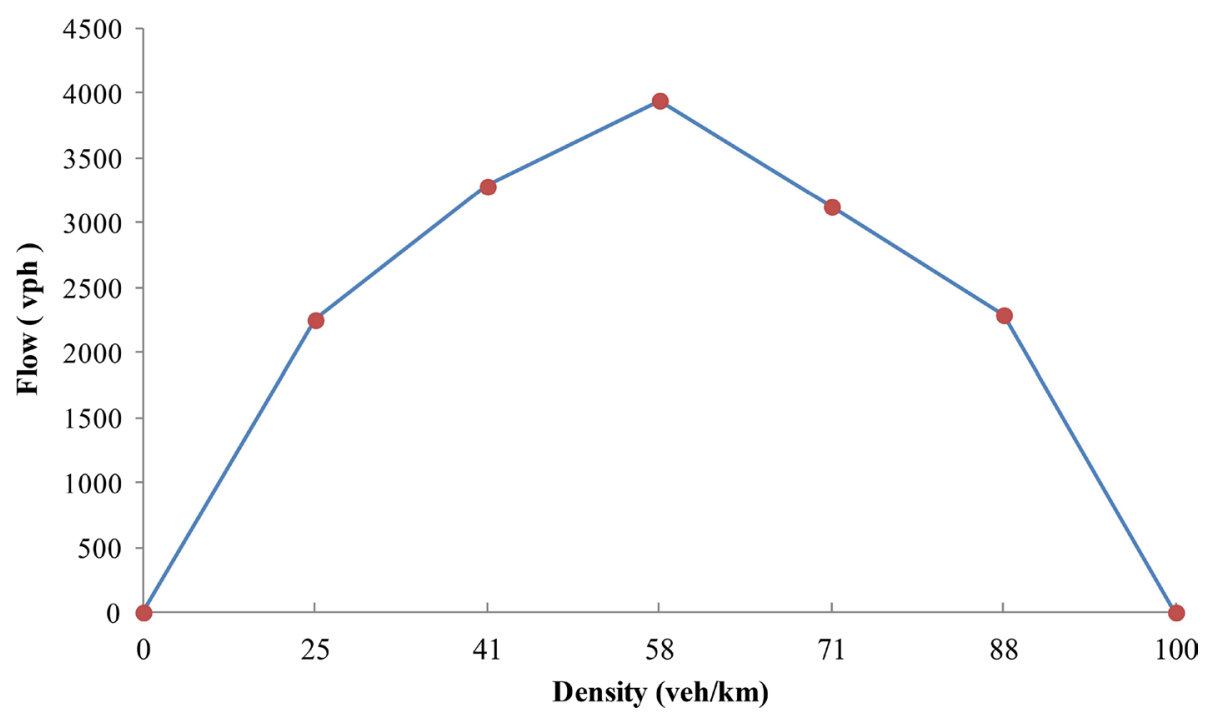

Fig. 10 The fundamental diagram used in this experiment

Table 1 The samples of densityvelocity-flow relationship in a highway

\begin{tabular}{llllllll}
\hline Density (veh/km) & 0 & 25 & 41 & 58 & 71 & 88 & 100 \\
\hline Velocity $(\mathrm{km} / \mathrm{h})$ & 100 & 90 & 80 & 68 & 44 & 26 & 0 \\
Flow $(\mathrm{veh} / \mathrm{h})$ & 0 & 2250 & 3280 & 3944 & 3124 & 2288 & 0 \\
\hline
\end{tabular}

length in the CTM is $2 \mathrm{~km}$ and the sampling period is $0.02 \mathrm{~h}$. The fundamental diagram of this road is a triangle. The free velocity, best density, and jam density are $100 \mathrm{~km} / \mathrm{h}, 39.44$ $\mathrm{veh} / \mathrm{km}$, and $100 \mathrm{veh} / \mathrm{km}$, respectively. Initial flows on the road are set as $3944 \mathrm{vph}(\mathrm{veh} / \mathrm{h})$. Dynamic flows for these two cases are different. Case-I: The inflow onto the entry and the on-ramp flow are $2000 \mathrm{vph}$ and $1000 \mathrm{vph}$ in the 100th minute, respectively; Case-II: the flows before the 135th minute are the same as in Case-I, but the on-ramp flow is changed to $1500 \mathrm{vph}$ in the 135 th minute.

As the CTM is influenced by the cell length, it is difficult to say whether the on-ramp is connected to the 29th or 30th cell when compared with the RLWR-RSM. Therefore, both of them are tested. Besides, travel times are determined for two kinds of states. (1) Instant state: the traffic state in a road at a particular moment remains unchanged and the travel time for a vehicle to pass this road is calculated. (2) Evolutional state: considering the evolution of traffic states, dynamic velocities depend on the instantaneous traffic state and vehicle's position at current moment. In Fig. 22, the RLWR-SRM considers the evolutional state, and the other models consider the instant state; the on-ramps in CTM1-Instant and CTM2-Instant are located in the 29th and 30th cells, respectively. 


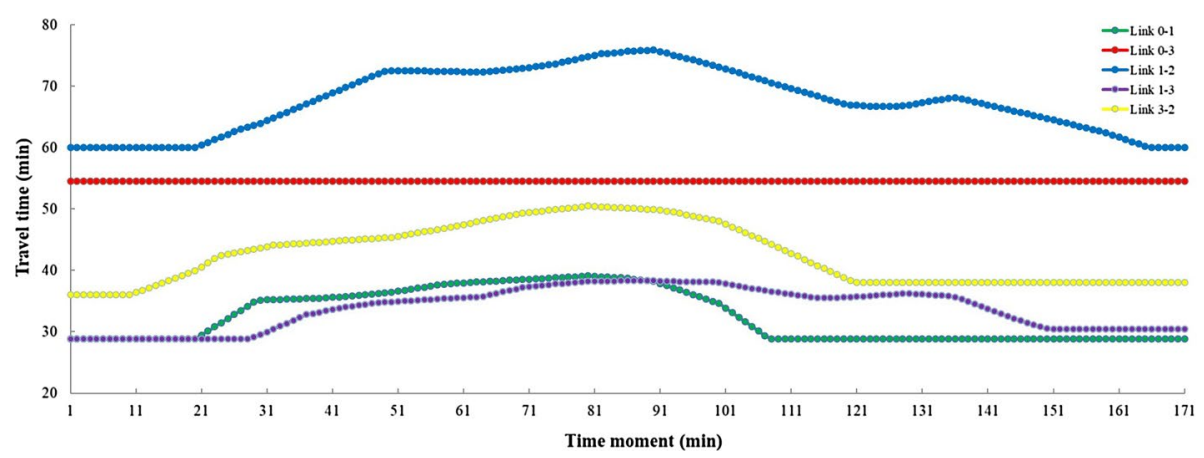

Fig. 11 The estimated road travel times under time-varying OD matrix with 10-min sampling period

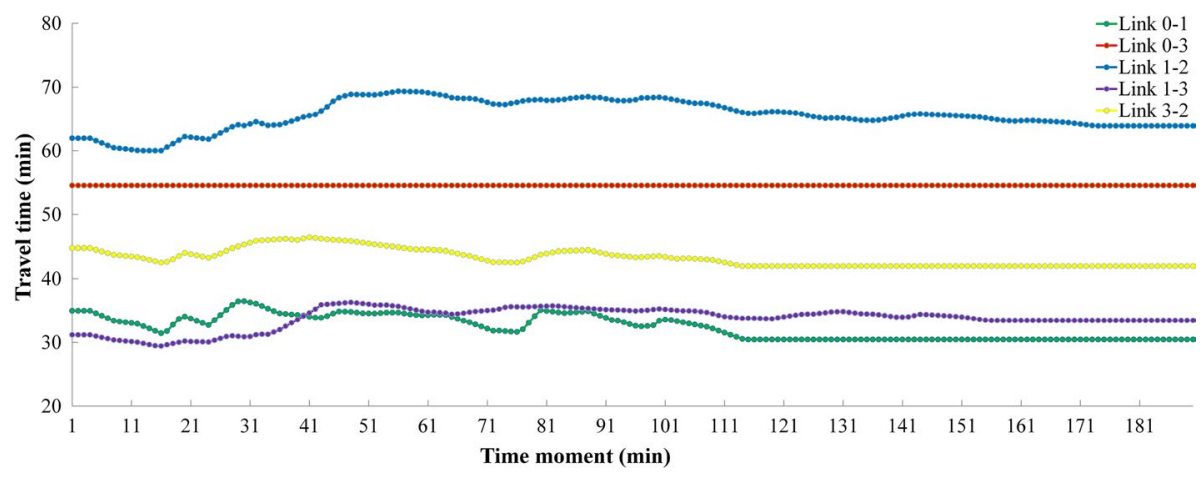

Fig. 12 The estimated road travel times under time-varying OD matrix with 4-min sampling period

\section{Result and discussion}

In order to understand how disturbances affect the traffic flows in a network, the travel times under each disturbance are estimated independently and together. Moreover, several 'unusual' phenomena are explained using this hybrid model.

(1) Travel times under time-varying OD matrix Figures 11 and 12 show the estimated dynamic travel times affected by the time-varying OD matrix. The changing tendencies of dynamic travel times and corresponding time-varying OD matrix are similar. Experimental results demonstrate the following: (1) All curve gradients are larger than -1 . For two neighbouring vehicles $\chi_{1}$ and $\chi_{2}$, let the time moments at the entry be $t_{0}$ and $t_{0}+1$, respectively; then, their travel times $T_{0}$ and $T_{1}$ satisfy $T_{0}+t_{0}<T_{1}+t_{0}+1$. Thus, the first-in-first-out (FIFO) characteristic is followed; overtaking is not allowed in these cases. (2) In the incipient and anaphase phases, the travel times are constant because the traffic flow remains unchanged and the vehicles are in equilibrium states without encountering any shockwave. Thus, the 'reduction to static model' characteristic is also followed. (3) No vehicle loss or addition verifies the 'flow conservation' characteristic. (4) From the above derivation of a vehicle's dynamic velocities, the travel times on a road are affected only by the vehicles in front of it and not by those behind 
it. This reveals the 'causality' principle. (5) In Figs. 11 and 12, no vehicle goes onto road $(0,3)$ because it is not a route for an arbitrary pair of OD; the traffic states on road $(3,2)$ are only affected by the vehicles from node 3 to node 2 ; multiple OD vehicles travel on the same road. For example, both OD $(0,2)$ and $(1,2)$ affect the traffic states of road $(1,2)$. These contributions can be attributed to the RSM, which allows the RLWR-RSM to model dynamic traffic flows not only for individual roads but also for the entire network. (6) The main difference between "Appendix 1 and 2" is the sampling period of the OD matrix. Corresponding travel times in Figs. 11 and 12 fluctuate at different frequencies.

(2) Travel times under dynamic disruptions Figure 13 shows the estimated road travel times for the static OD matrix ("Appendix 3") with fixed route choices, but under a disruption. From Fig. 13, the curves of travel times on roads $(1,2)$ and $(1,3)$ show similar tendencies: (1) At the 41st minute, sudden jumps are observed in the curves; this is because a vehicle at this time moment begins to experience congestion, whereas those vehicles in front of it do not. (2) After the 41st minute, on one hand, the congested shockwaves move upstream, causing a prolonged congestion. On the other hand, the disruption recovery mitigates the effects of congestion; these two factors work together. The travel times initially increase and then decrease. In earlier periods, predominant congested shockwaves increase the vehicles' travel times, but the disruption recovery plays a more important role in the later periods. (3) The travel times before the occurrence and after the disappearance of all disruptions on roads $(1,2)$ and $(1,3)$ are different. This is because only $O D(1,2)$ or $O D(1,3)$ vehicles travel on roads $(1,2)$ and $(1,3)$ before ripples appear, but $O D(0,3)$ or $O D(0,2)$ vehicles are on the roads even after all ripples disappear. (4) Figure 14 can be considered as an example to explain the change in traffic flows. The traffic state is in the equilibrium state 3 if no disturbance exists. When a disruption arises, two new states (i.e., states 1 and 8) are located at the upstream and downstream directions of the disruption, and they move upstream and downstream, respectively. Subsequently, due to disruption recovery, the traffic state at the upstream of disruption changes from state 8 to state 7 , state 6 , and state 5 in turn. The traffic state at the downstream of disruption changes from state 1 to state 2 , state 4 , and state 5 in turn. These states propagate upstream and downstream, respectively. The disruption disappears when the traffic states at the upstream and downstream directions are the same (state 5). Not only disruptions but also inflows affect the traffic states. When the traffic state at the entry changes to state 6 or state 5, a new shockwave is generated in the entry and affects the road travel

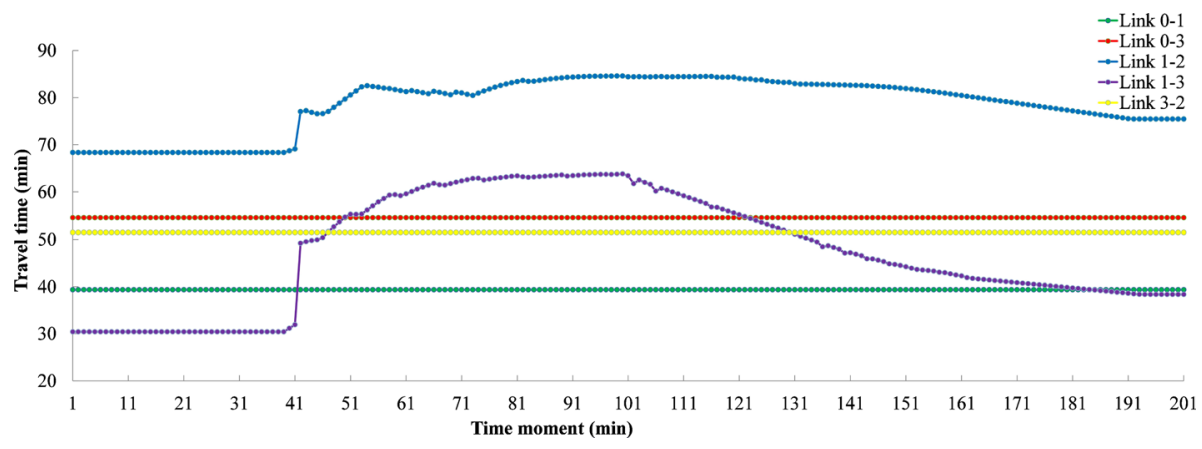

Fig. 13 The estimated road travel times under road disruptions 


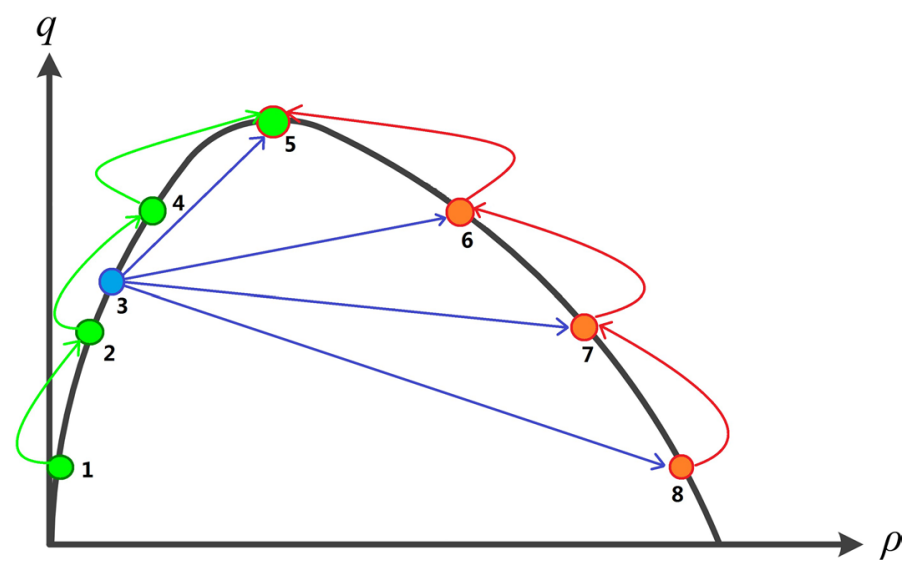

Fig. 14 The changing traffic states considering the generation and recovery of road disruptions

times if the inflows from the OD matrix and outflows from incoming roads correspond to state 3 . In addition, the inflow of $(1,2)$ or $(1,3)$ also changes not only because of the disruption but also because of dynamic flows of $O D(0,3)$ or $O D(0,2)$.

(3) Travel times under dynamic route choices Experimental results shown in Figs. 15 and 16 demonstrate the following: (1) The inflows onto roads $(0,1)$ and $(0,3)$ are complementary because of the flow conservation. (2) Inflow onto road $(1,2)$ shows a general downward trend, but a momentary jump appears at the 39th minute because of the outflows from OD $(0,2)$. (3) Inflows onto roads $(1,3)$ and $(3,2)$ persistently increase. The corresponding estimated travel times are given in Fig. 16. It must be mentioned that the travel times on roads $(0,3)$ and $(3,2)$ remain constant although their inflows change by a small value. Small disturbances in a free road will not decrease the travel times.

(4) Travel times under mixed disturbances As mentioned above, three kinds of disturbances are independently studied. Moreover, the hybrid RLWR-RSM can model the dynamic traffic flows and estimate dynamic road travel times under simultaneous multiple disturbances. Figures 17 and 18 respectively show the estimated travel times

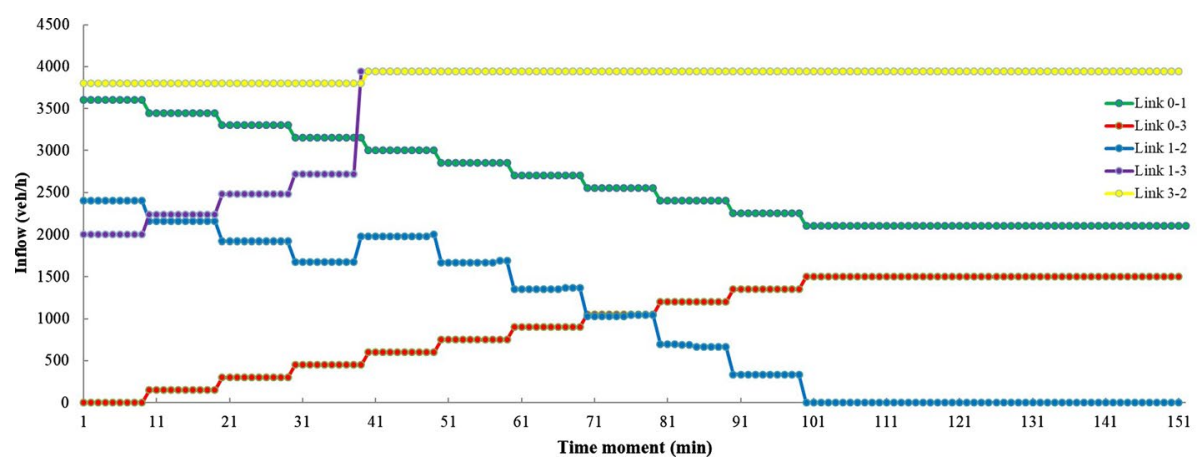

Fig. 15 The dynamic inflows considering dynamic route choices 


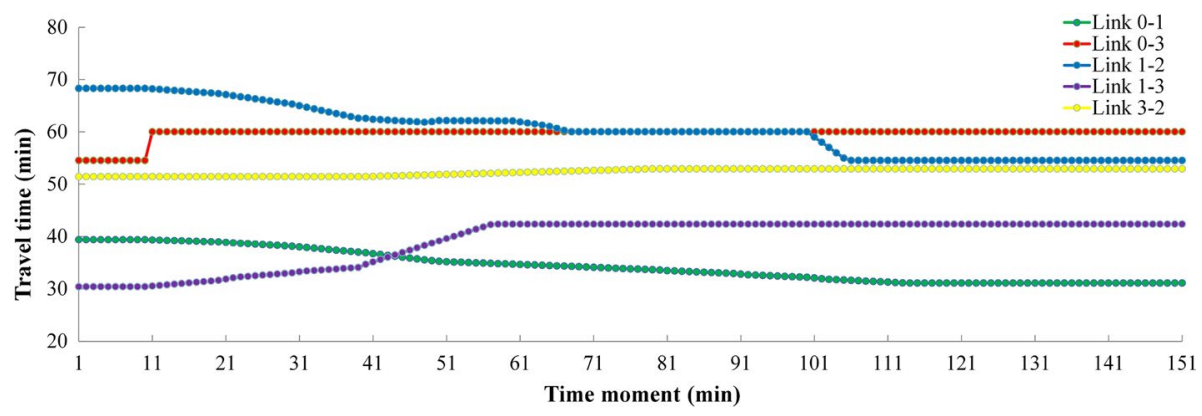

Fig. 16 The estimated road travel times considering dynamic route choices

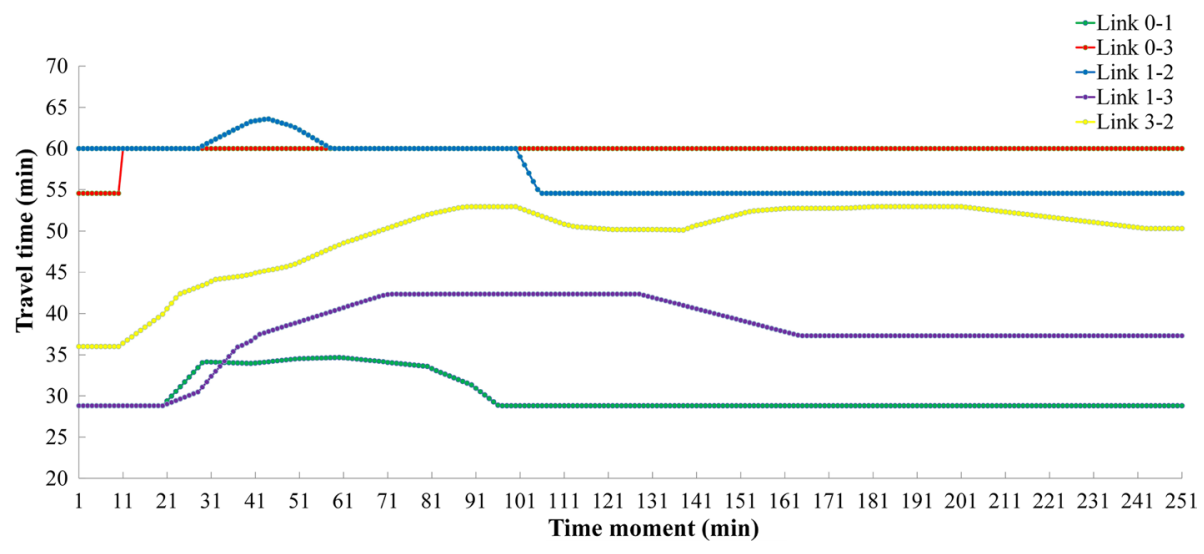

Fig. 17 The estimated road travel times considering dynamic OD matrix and route choices

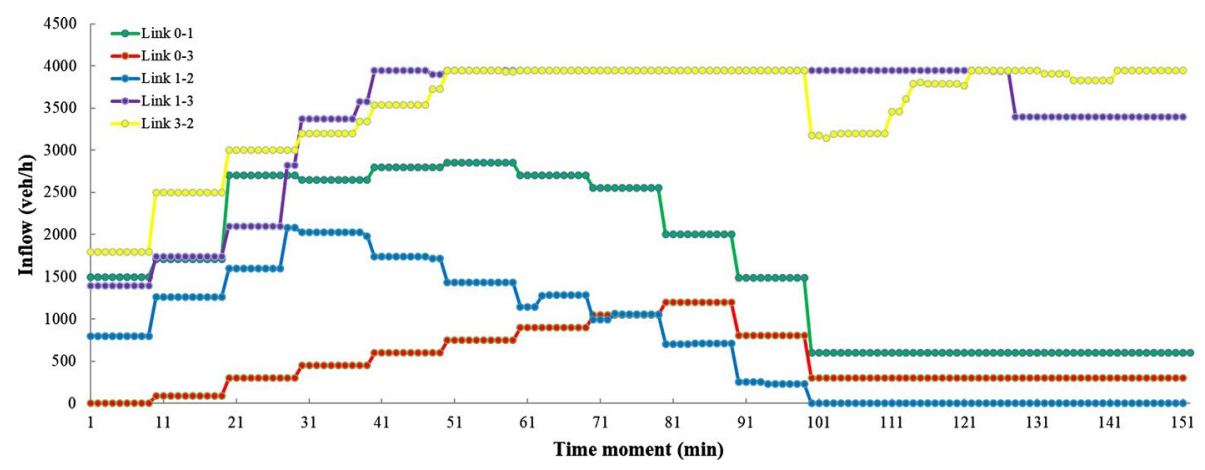

Fig. 18 The dynamic inflows considering dynamic OD matrix and route choices

and inflows onto roads considering the dynamic OD matrix ("Appendix 1") and route choices. Both of them affect the traffic flows by changing the flow distribution at the exit of the road. The travel times depend on inflows (Fig. 17) and sometimes on outflows when the outgoing road/roads is/are under congestion. If all disturbances are considered together, the situation becomes more complicated, as shown in Figs. 19 and 20. 


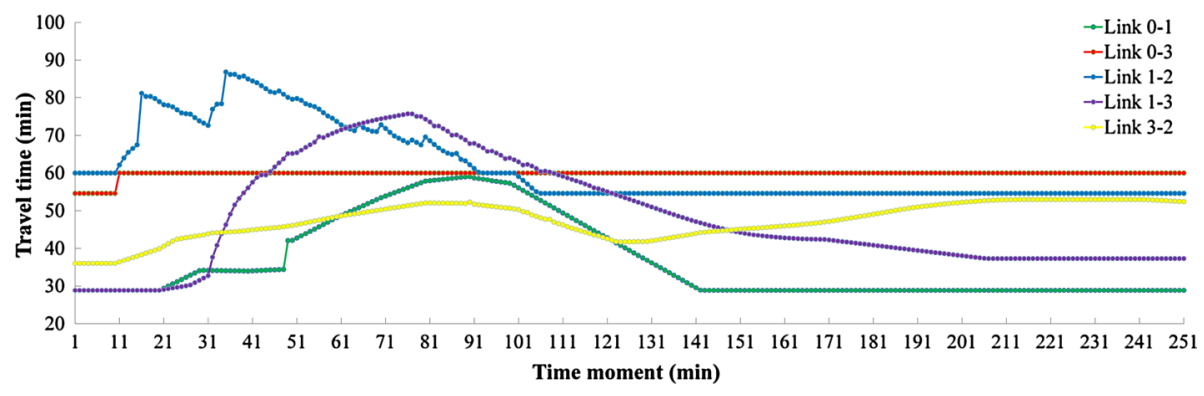

Fig. 19 The estimated road travel times considering dynamic OD matrix, route choices, and road disruptions

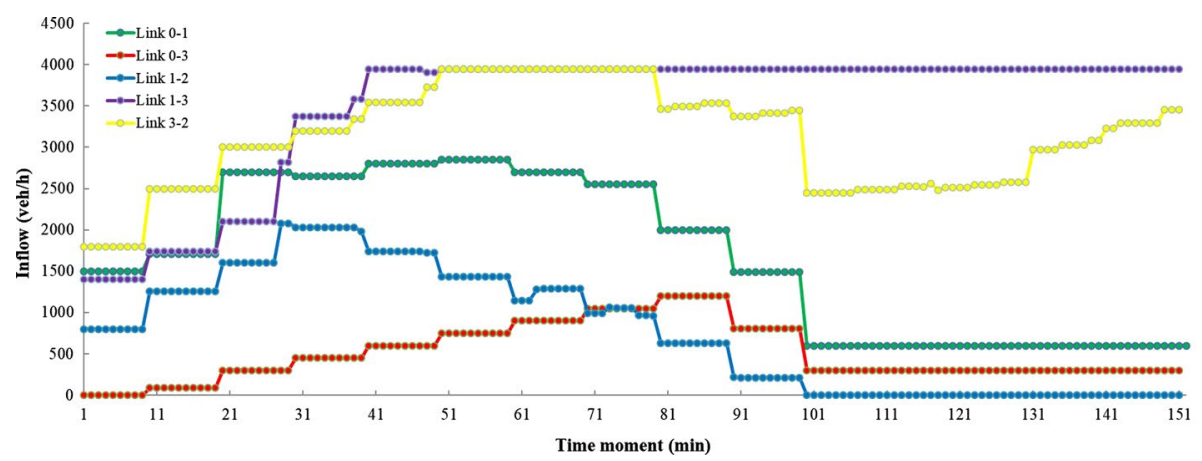

Fig. 20 The dynamic inflows considering dynamic OD matrix, route choices, and road disruptions

The outflow/outflows of an incoming road/roads of $i$ may affect the travel times on another incoming road of $i$. For example, if more flows reach the exit of road $(1,3)$ and road $(3,2)$ is under congestion, the maximum outflow of road $(0,3)$ decreases and a new upward shockwave is generated at the exit of road $(0,3)$. Regardless of how congestion and congestion-recoveries spread over the entire network, both the vehicle conservation and drivers' choices are retained.

(5) Explanation of 'unusual' phenomena Some of the literature has mentioned several 'unusual' phenomena in real-world traffic flows. For example, Kerner and Rehborn (1997) empirically showed that local traffic congestion persists for several hours while maintaining its form and characteristic properties. In light of the RLWR model, the speed of the shockwave is zero if the flow rates at the upstream and downstream directions are the same, but densities are different. The local congestion neither spreads nor vanishes. This situation is the same with the disruption situation without recoveries in the RLWR-RSM. The presented case (where shockwave $v_{c}=0$ ) in the RLWR-RSM can explain this interesting phenomenon well.

Another phenomenon is that a disturbance propagates from one vehicle to the next and is amplified until a vehicle comes to a complete stop at some point. This situation is similar to the case $d$ in Fig. 4, but the values of $v_{1}$ and $v_{2}$ are different, as shown in Fig. 21. According to the aforementioned three stages, the vehicles' velocities decrease one by one 


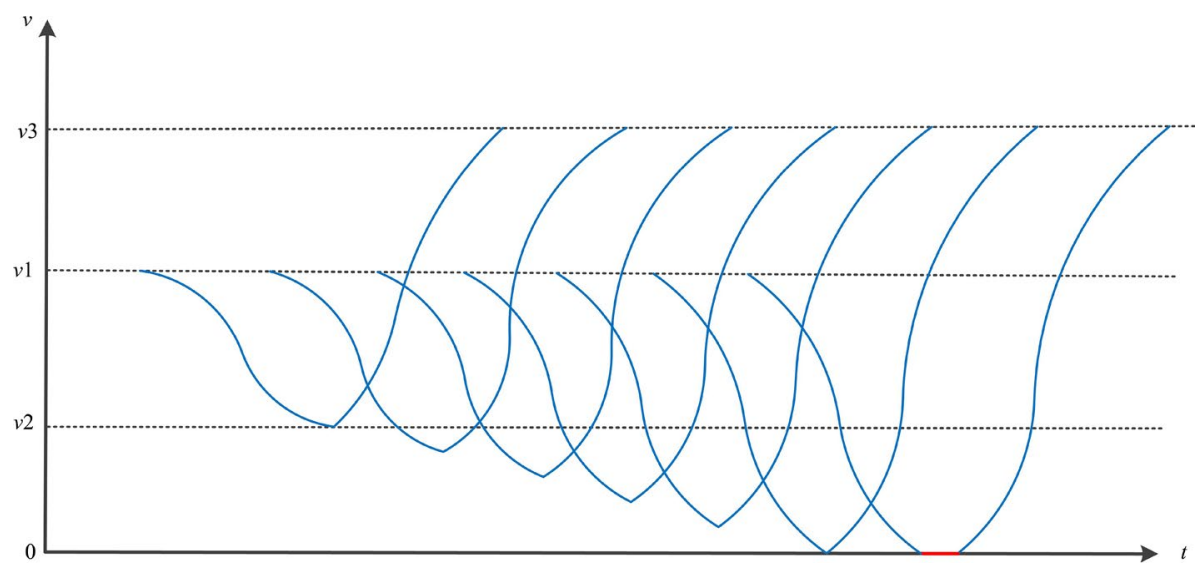

Fig. 21 The vehicles' behaviors when a disturbance occurs in a serious traffic state

to maintain a safe distance. The traffic flows are under total congestion when the dynamic process evolves to Stage-3.

(6) Comparison with the CTM Comparing the RLWR-RSM with the CTM in an instant state (Fig. 22), all the trends are the same, but some extreme values are different when congestion propagates along the road. In Case-I, RLWR-RSM-Instant is very close to CTM1Instant at most of times, except for the case when two shockwaves interact. At this moment, the performance of RLWR-RSM-Instant is similar to that of CTM2-Instant. In Case-II, the travel time curve of CTM1-Instant bends earlier than that of RLWR-RSM-Instant at the 122th minute and later at approximately the 135th and 143rd minutes, while the travel time curve of CTM2-Instant bends earlier than that of RLWR-RSM-Instant at approximately the 122th and 135th minutes, and later at approximately the 143rd minute. This indicates the following: (1) The first shockwave merge process in the CTM occurs faster than that in the RLWR-RSM, but the second and third ones are slow to occur. (2) The difference between the RLWR-RSM and CTM becomes bigger when more shockwaves are encountered. (3) The shockwave merge process in the CTM is more chaotic than that in the RLWR-RSM. Thus, it is difficult to decide which one is better. However, it can be said that the presence of cells creates a deviation in the results of the CTM because of the inaccurate location of the on-ramp.

Comparing the instant and evolutional states, the travel times of RLWR-RSM and RLWR-RSM-Instant are entirely different. The RLWR-RSM considers the predicted future traffic states, whereas other models do not. For example, a vehicle that enters a road at the 64th minute may meet with the first shockwave in the future (e.g., at the 100th minute). As a result, its travel times will be long. The RLWR-RSM describes this better than the RLWR-RSM-Instant and the CTM. Thus, the RLWR-RSM is more practical, which is one of our contributions. 


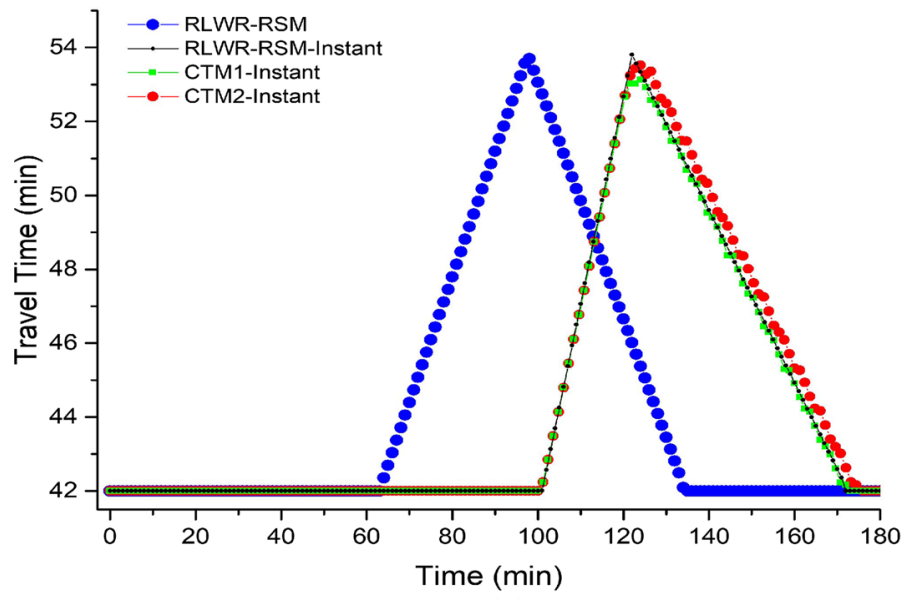

(a)

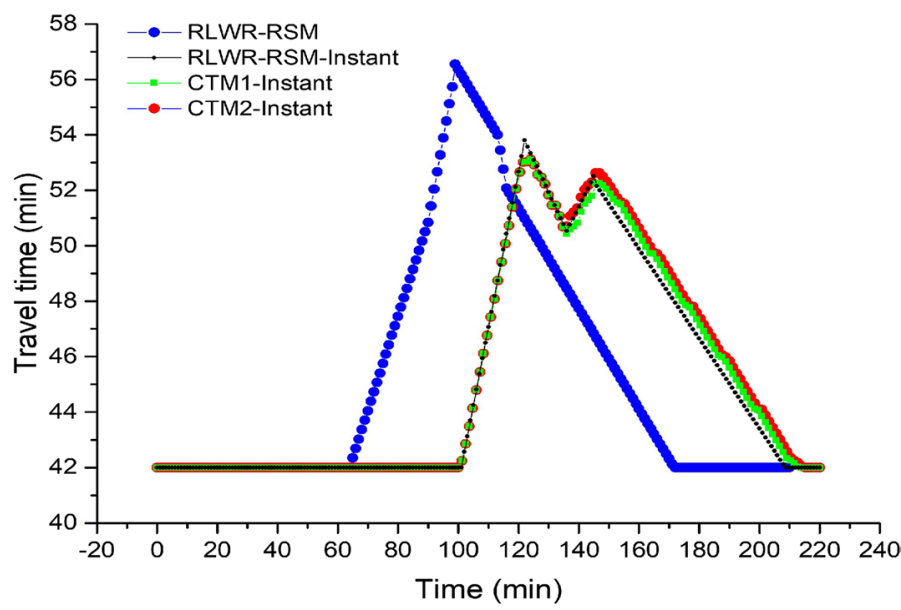

(b)

Fig. 22 The estimated travel times compared with the CTM model: (a) under one disturbance; (b) under two disturbances, where RLWR-RSM consideres the evolution of dynamic traffic states for each specific vehicle, while RLWR-RSM-Instant, CTM1-Instant, and CTM2-Instant don't

\section{Concluding remarks}

In this paper, we proposed a hybrid RLWR-RSM to model dynamic traffic flows and estimate the road travel times at arbitrary time moments in a transportation network under a time-varying OD matrix, dynamic route choices, and dynamic disruptions. This hybrid model inherits the advantages of generic macroscopic traffic flow models and maintains vehicles' characteristics by integrating the revised Lighthill-Whitham-Richards and ripple-spreading models. The limitation of the conventional LWR model where non-equilibrium states cannot be described was overcome by considering a buffer for vehicles to gradually change their velocities. Furthermore, the interactions of different traffic states not only within individual roads but also in an entire network were investigated using the hybrid RLWR-RSM. Four basic properties (FIFO, causality, reduction to static model, and 
flow conversation) of traffic flows were demonstrated and confirmed by experiments with various inputs. In addition, two common but special phenomena mentioned in the literature were explained by the RLWR-RSM.

With the development of location positioning technologies (such as GPS), floating car data with real geographic position and travel speed can be obtained in a relatively easy way. Existing applications generally utilise only the travel speed of vehicles for the estimation of congestion and travel times (Lim et al. 2016; Tong et al. 2013). However, modelling of dynamic traffic flows from pure and sparse observation data remains challenging (Arbués and Baños 2016) although video monitoring systems located at intersections can provide limited valuable information on dynamic traffic inflows (or outflows) onto (or off) a road. One reason for this is that theoretical traffic flow models and the valuable realistic data have not been adequately integrated with each other. Using the contributions of this paper, high-precision practical traffic flows can be estimated and predicted when coupled with current advanced observation data, such as GPS, surveillance video, and magnetic vehicle detector data. As a result, quantitative and high-efficiency emergency vehicle evacuation and dynamic congestion alleviation can be achieved. In the future, we will collect related realistic data, determine the flow-density relationship, and capture drivers' (driverless') behavioural characteristics. Using these data, the RLWR-RSM with related explicit parameters can be compared to realistic dynamic traffic flows and travel times so that the RLWR-RSM can be widely used in transportation management and emergency response applications. Moreover, the proposed hybrid RLWR-RSM can be used to optimise driverless vehicle behaviours.

Acknowledgements This work was supported by the National Natural Science Foundation of China: [Grant Numbers 41701452]; The National Key Research and Development Program of China (2017YFB0504203); The Strategic Priority Research Program of the Chinese Academy of Sciences (No. XDA19030301).

\section{Compliance with ethical standards}

Conflict of interest On behalf of all authors, the corresponding author states that there is no conflict of interest.

\section{Appendix 1: The time-varying OD matrix in which the sampling period is 10 minute}

See Table 2 .

Table 2 The time-varying OD matrix in which the sampling period is 10 minutes

\begin{tabular}{llllllllllll}
\hline OD flow (vph) & \multicolumn{1}{l}{ Time(min) } \\
\cline { 2 - 10 } & 0 & 10 & 20 & 30 & 40 & 50 & 60 & 70 & 80 & 90 & 100 \\
\hline $0-1$ & 300 & 300 & 600 & 700 & 700 & 900 & 1200 & 1200 & 900 & 600 & 300 \\
$0-2$ & 600 & 600 & 900 & 900 & 1200 & 1200 & 900 & 900 & 800 & 800 & 300 \\
$0-3$ & 600 & 900 & 1500 & 1500 & 1500 & 1500 & 1500 & 1500 & 1500 & 900 & 300 \\
$1-3$ & 1400 & 1600 & 1700 & 1900 & 2000 & 2200 & 2400 & 2200 & 2000 & 1900 & 1700 \\
$1-2$ & 800 & 1400 & 2000 & 2300 & 2300 & 2300 & 2300 & 2400 & 2400 & 1400 & 1400 \\
$3-2$ & 1800 & 2500 & 3000 & 3200 & 3400 & 3800 & 3900 & 3800 & 3200 & 3000 & 2000 \\
\hline
\end{tabular}




\section{Appendix 2: The time varying OD matrix in which the sampling period is 4 minutes}

See Table 3.

Table 3 The time varying OD matrix in which the sampling period is 4 minutes

\begin{tabular}{|c|c|c|c|c|c|c|c|c|c|c|c|c|c|}
\hline \multirow[t]{3}{*}{ OD flow (vph) } & \multicolumn{13}{|c|}{ Time(min) } \\
\hline & 0 & 4 & 8 & 12 & 16 & 20 & 24 & 28 & 32 & 36 & 40 & 44 & 48 \\
\hline & 52 & 56 & 60 & 64 & 68 & 72 & 76 & 80 & 84 & 88 & 92 & 96 & 100 \\
\hline \multirow[t]{2}{*}{$0-1$} & 1013 & 596 & 930 & 618 & 1299 & 572 & 1210 & 1029 & 711 & 1087 & 979 & 1021 & 939 \\
\hline & 1047 & 917 & 979 & 687 & 599 & 912 & 1249 & 812 & 1005 & 640 & 848 & 992 & 670 \\
\hline \multirow[t]{2}{*}{$0-2$} & 713 & 496 & 630 & 518 & 899 & 472 & 810 & 729 & 611 & 787 & 679 & 721 & 739 \\
\hline & 747 & 617 & 779 & 587 & 499 & 712 & 849 & 612 & 705 & 440 & 648 & 792 & 570 \\
\hline \multirow[t]{2}{*}{$0-3$} & 1213 & 696 & 1030 & 718 & 1399 & 672 & 1310 & 1129 & 811 & 1187 & 1079 & 1221 & 1039 \\
\hline & 1147 & 1017 & 1079 & 787 & 599 & 1012 & 1349 & 912 & 1105 & 640 & 948 & 1192 & 770 \\
\hline \multirow[t]{2}{*}{$1-2$} & 1913 & 1196 & 1730 & 1418 & 2199 & 1272 & 2110 & 1929 & 1511 & 1987 & 1779 & 2021 & 1739 \\
\hline & 1847 & 1717 & 1879 & 1487 & 1199 & 1812 & 2149 & 1512 & 1805 & 1240 & 1548 & 1892 & 1470 \\
\hline \multirow[t]{2}{*}{$1-3$} & 2113 & 1696 & 1930 & 1718 & 2299 & 1672 & 2210 & 2129 & 1811 & 2187 & 2079 & 2121 & 2039 \\
\hline & 2047 & 2017 & 2079 & 1787 & 1699 & 2012 & 2249 & 1812 & 2005 & 1740 & 1848 & 2092 & 1770 \\
\hline \multirow[t]{2}{*}{$3-2$} & 3213 & 2396 & 2930 & 2518 & 3599 & 2372 & 3510 & 3229 & 2711 & 3287 & 3079 & 3321 & 3039 \\
\hline & 3147 & 3017 & 3079 & 2587 & 2299 & 3012 & 3549 & 2712 & 3105 & 2440 & 2748 & 3192 & 2570 \\
\hline
\end{tabular}

\section{Appendix 3: The static OD matrix}

See Table 4.

Table 4 The static OD matrix

\begin{tabular}{lllllll}
\hline OD & $0-1$ & $0-2$ & $0-3$ & $1-2$ & $1-3$ & $3-2$ \\
\hline flow(vph) & 1200 & 900 & 1500 & 2000 & 2400 & 3800 \\
\hline
\end{tabular}

\section{Appendix 4: The route path matrix}

See Table 5.

Table 5 The route path matrix, where the elements indicate the next node of $P_{O, D}^{k}$

\begin{tabular}{lllllll}
\hline OD & $0-1$ & $0-2$ & $0-3$ & $1-2$ & $1-3$ & $3-2$ \\
\hline$p_{1}$ & 1 & 1 & 1 & 2 & 3 & 2 \\
$p_{2}$ & 1 & 1 & 1 & 3 & 3 & 2 \\
$p_{3}$ & 1 & 1 & 3 & 2 & 3 & 2 \\
\hline
\end{tabular}




\section{Appendix 5: The route choices}

See Table 6.

Table 6 The route choices, where the elements indicate the percentages of vehicles choosing $P_{O, D}^{k}$ at particular time moments

\begin{tabular}{lllllllllllll}
\hline $\begin{array}{l}\text { Path choice } \\
\text { (\%) }\end{array}$ & \multicolumn{1}{l}{ Time(min) } \\
\cline { 2 - 6 } & 0 & 10 & 20 & 30 & 40 & 50 & 60 & 70 & 80 & 90 & 100 \\
\hline OD 0-1 & $p_{1}$ & 100 & 90 & 80 & 70 & 60 & 50 & 40 & 30 & 20 & 10 & 0 \\
& $p_{2}$ & 0 & 5 & 10 & 15 & 20 & 25 & 30 & 35 & 40 & 45 & 50 \\
& $p_{3}$ & 0 & 5 & 10 & 15 & 20 & 25 & 30 & 35 & 40 & 45 & 50 \\
OD 0-2 & $p_{1}$ & 100 & 80 & 60 & 40 & 20 & 0 & 10 & 30 & 50 & 70 & 90 \\
& $p_{2}$ & 0 & 10 & 20 & 30 & 40 & 50 & 45 & 35 & 25 & 15 & 5 \\
& $p_{3}$ & 0 & 10 & 20 & 30 & 40 & 50 & 45 & 35 & 25 & 15 & 5 \\
OD 0-3 & $p_{1}$ & 100 & 90 & 80 & 70 & 60 & 50 & 40 & 30 & 20 & 10 & 0 \\
& $p_{2}$ & 0 & 5 & 10 & 15 & 20 & 25 & 30 & 35 & 40 & 45 & 50 \\
& $p_{3}$ & 0 & 5 & 10 & 15 & 20 & 25 & 30 & 35 & 40 & 45 & 50 \\
OD 1-3 & $p_{1}$ & 100 & 80 & 60 & 40 & 20 & 0 & 10 & 30 & 50 & 70 & 90 \\
& $p_{2}$ & 0 & 10 & 20 & 30 & 40 & 50 & 45 & 35 & 25 & 15 & 5 \\
& $p_{3}$ & 0 & 10 & 20 & 30 & 40 & 50 & 45 & 35 & 25 & 15 & 5 \\
OD 1-2 & $p_{1}$ & 100 & 90 & 80 & 70 & 60 & 50 & 40 & 30 & 20 & 10 & 0 \\
& $p_{2}$ & 0 & 5 & 10 & 15 & 20 & 25 & 30 & 35 & 40 & 45 & 50 \\
& $p_{3}$ & 0 & 5 & 10 & 15 & 20 & 25 & 30 & 35 & 40 & 45 & 50 \\
OD 3-2 & $p_{1}$ & 100 & 80 & 60 & 40 & 20 & 0 & 10 & 30 & 50 & 70 & 90 \\
& $p_{2}$ & 0 & 10 & 20 & 30 & 40 & 50 & 45 & 35 & 25 & 15 & 5 \\
& $p_{3}$ & 0 & 10 & 20 & 30 & 40 & 50 & 45 & 35 & 25 & 15 & 5 \\
\hline
\end{tabular}

\section{References}

Arbués, P., Baños, J.F.: A dynamic approach to road freight flows modeling in spain. Transportation 43(3), 549-564 (2016)

Carey, M., Ge, Y.E.: Comparing whole-link travel time models. Transp. Res. Part B Methodol. 37(10), 905-926 (2003)

Carey, M., Ge, Y.E., McCartney, M.: A whole-link travel-time model with desirable properties. Transp. Sci. 37(1), 83-96 (2003)

Castillo, E., Jiménez, P., Menéndez, J.M., Nogal, M.: A bayesian method for estimating traffic flows based on plate scanning. Transportation 40(1), 173-201 (2013)

Cho, H.J., Lo, S.C.: Modeling self-consistent multi-class dynamic traffic flow. Physica A Stat. Mech. Appl. 312(3), 342-362 (2002)

Daganzo, C.F.: The cell transmission model: a dynamic representation of highway traffic consistent with the hydrodynamic theory. Transp. Res. Part B Methodol. 28(4), 269-287 (1994)

Daganzo, C.F.: The cell transmission model: a simple dynamic representation of highway traffic. Transp. Res. Part B Methodol. 28(4), 269-287 (1994)

Daganzo, C.F.: The cell transmission model, part II: network traffic. Transp. Res. Part B Methodol. 29(2), 79-93 (1995)

Daganzo, C.F.: Requiem for second-order fluid approximations of traffic flow. Transp. Res. Part B Methodol. 29(4), 277-286 (1995)

$\mathrm{Fu}$, L.: Scheduling dial-a-ride paratransit under time-varying, stochastic congestion. Transp. Res. Part B Methodol. 36(6), 485-506 (2002)

Fu, L., Rilett, L.R.: Expected shortest paths in dynamic and stochastic traffic networks. Transp. Res. Part B Methodol. 32(7), 499-516 (1998) 
Gentile, G., Meschini, L., Papola, N.: Spillback congestion in dynamic traffic assignment: a macroscopic flow model with time-varying bottlenecks. Transp. Res. Part B Methodol. 41(10), 1114-1138 (2007)

Helbing, D.: Gas-kinetic derivation of navier-stokes-like traffic equations. Phys. Rev. E 53(3), 2366 (1996)

Hirsch, H.: Numerical Computation of Internal and External Flows: The Fundamentals of Computational Fluid Dynamics. Wiley, New York (1990)

Hoogendoorn, S., van Lint, H., Knoop, V.: Macroscopic modeling framework unifying kinematic wave modeling and three-phase traffic theory. Transp. Res. Rec. J. Transp. Res. Board 2088, 102-108 (2008)

Hoogendoorn, S.P., Bovy, P.H.: State-of-the-art of vehicular traffic flow modelling. Proc. Inst. Mech. Eng. Part I J. Syst. Control Eng. 215(4), 283-303 (2001)

Hu, X.B., Wang, M., Leeson, M.S., Hines, E.L., Di Paolo, E.: Deterministic ripple-spreading model for complex networks. Phys. Rev. E 83(4), 046123 (2011)

Jiang, J., Li, Q., Wu, L., Tu, W.: Multi-objective emergency material vehicle dispatching and routing under dynamic constraints in an earthquake disaster environment. ISPRS Int. J. Geo Inf. 6(5), 142 (2017)

Jiang, J., Wu, L.: A new dynamic network flow algorithm using base state amendment model for emergency response. Trans. GIS 21(6), 1179-1203 (2017)

Jin, W.L.: A kinematic wave theory of lane-changing traffic flow. Transp. Res. Part B Methodol. 44(8), 10011021 (1977)

Kerner, B.S., Rehborn, H.: Experimental properties of phase transitions in traffic flow. Phys. Rev. Lett. 79(20), 4030 (1997)

Kim, S., Lewis, M.E., White, C.C.: Optimal vehicle routing with real-time traffic information. IEEE Trans. Intell. Transp. Syst. 6(2), 178-188 (2005)

Kockelman, K.M.: Modeling traffic's flow-density relation: accommodation of multiple flow regimes and traveler types. Transportation 28(4), 363-374 (2001)

Lighthill, M.J., Whitham, G.B.: On kinematic waves. II. a theory of traffic flow on long crowded roads. Proc. R. Soc. London A: Math. Phys. Eng. Sci. 229(1178), 317-345 (1955)

Lim, S.H., Kim, Y., Lee, C.: Real-time travel-time prediction method applying multiple traffic observations. KSCE J. Civ. Eng. 20(7), 2920-2927 (2016)

Liu, G., Lyrintzis, A., Michalopoulos, P.: Improved high-order model for freeway traffic flow. Transp. Res. Rec. J. Transp. Res. Board 1644, 37-46 (1998)

Lnnamaa, S.: Short-term prediction of travel time using neural networks on an interurban highway. Transportation 32(6), 649-669 (2005)

Matas, A., Raymond, J., Ruiz, A.: Traffic forecasts under uncertainty and capacity constraints. Transportation 39(1), 1-17 (2012)

Michalopoulos, P.G., Yi, P., Lyrintzis, A.S.: Development of an improved high-order continuum traffic flow model. Transp. Res. Rec. 1365, 485-498 (1992)

Newell, G.F.: Nonlinear effects in the dynamics of car following. Oper. Res. 9(2), 209-229 (1961)

Papageorgiou, M., Diakaki, C., Dinopoulou, V., Kotsialos, A., Wang, Y.: Review of road traffic control strategies. Proc. IEEE 91(12), 2043-2067 (2003)

Payne, H.J.: Models of freeway traffic and control. Math. Models Public Syst. 1(28), 51-61 (1971)

Payne, H.J.: Freflo: a macroscopic simulation model of freeway traffic. Transp. Res. Rec. 722, 68-72 (1979)

Peeta, S., Ziliaskopoulos, A.K.: Foundations of dynamic traffic assignment: the past, the present and the future. Netw. Spat. Econ. 1(3-4), 233-265 (2001)

Richards, P.I.: Shock waves on the highway. Oper. Res. 4(1), 42-51 (1956)

Sever, D., Dellaert, N., Van Woensel, T., De Kok, T.: Dynamic shortest path problems: hybrid routing policies considering network disruptions. Comput. Oper. Res. 40(12), 2852-2863 (2013)

Sheu, J.B., Chou, Y.H., Shen, L.J.: A stochastic estimation approach to real-time prediction of incident effects on freeway traffic congestion. Transp. Res. Part B Methodol. 35(6), 575-592 (2001)

Tong, D., Lin, W.H., Stein, A.: Integrating the directional effect of traffic into geostatistical approaches for travel time estimation. Int. J. Intell. Transp. Syst. Res. 11(3), 101-112 (2013)

Treiber, M., Kesting, A.: Traffic flow dynamics: data, models and simulation. Phys. Today 67(3), 54 (2014)

Turner, S.: Advanced techniques for travel time data collection. Transp. Res. Rec. J. Transp. Res. Board 1551, 51-58 (1996)

Whitham, G.B., Fowler, R.G.: Linear and nonlinear waves. Phys. Today 28(6), 55-56 (2008)

Xiong, C., Chen, X., He, X., Guo, W., Zhang, L.: The analysis of dynamic travel mode choice: a heterogeneous hidden markov approach. Transportation 42(6), 985-1002 (2015)

$\mathrm{Xu}, \mathrm{X}$., Chen, A., Cheng, L.: Assessing the effects of stochastic perception error under travel time variability. Transportation 40(3), 525-548 (2013)

Yeon, J., Elefteriadou, L., Lawphongpanich, S.: Travel time estimation on a freeway using discrete time markov chains. Transp. Res. Part B Methodol. 42(4), 325-338 (2008) 
Publisher's Note Springer Nature remains neutral with regard to jurisdictional claims in published maps and institutional affiliations.

Jincheng Jiang is an associate research professor of Shenzhen Institutes of Advanced Technology, Chinese Academy of Sciences. His research interests include traffic flow models, GIS, spatial-temporal data analysis, and emergency evacuation.

Nico Dellaert is an associate professor in Quantitative Modeling at the Technische Universiteit Eindhoven. His prime research interests are on transportation, healthcare logistics, and on vehicle routing with uncertainty.

Tom Van Woensel is a full professor of Freight Transport and Logistics in the OPAC group (Operations, Planning, Accounting and Control) of the department of Industrial Engineering and Innovation Sciences at Eindhoven University of Technology (TU/e). Current interests include last mile logistics, vehicle routing problems, crowd shipping, (service) network design, machine learning, big data and IoT-driven issues, and opportunities.

Lixin $\mathbf{W u}$ is the leading professor of Geomatics (Surveying and Mapping) in the School of Geoscience and Info-Physics of Central South University, China. His research interests include mainly geospatial informatics, disaster investigation, remote sensing, and geo-hazard perception. 